\title{
Angular momentum generation in nuclear fission
}

https://doi.org/10.1038/s41586-021-03304-w

Received: 18 June 2020

Accepted: 9 December 2020

Check for updates

\author{
J. N. Wilson ${ }^{1 凶}$, D. Thisse', M. Lebois', N. Jovančević', D. Gjestvang ${ }^{2}$, R. Canavan $^{3,4}$, \\ M. Rudigier ${ }^{3,5}$, D. Étasse ${ }^{6}$, R-B. Gerst ${ }^{7}$, L. Gaudefroy ${ }^{8}$, E. Adamska ${ }^{9}$, P. Adsley ${ }^{1}$, A. Algora ${ }^{10,11}$, \\ M. Babo', K. Belvedere ${ }^{3}$, J. Benito ${ }^{12}$, G. Benzoni ${ }^{13}$, A. Blazhev' ${ }^{7}$ A. Boso ${ }^{4}$, S. Bottoni ${ }^{13,14}$, \\ M. Bunce ${ }^{4}$, R. Chakma', N. Cieplicka-Oryńczak ${ }^{15}$, S. Courtin $^{16}$, M. L. Cortés ${ }^{17}$, P. Davies ${ }^{18}$, \\ C. Delafosse', M. Fallot ${ }^{19}$, B. Fornal ${ }^{15}$, L. Fraile ${ }^{12}$, A. Gottardo ${ }^{20}$, V. Guadilla $^{19}$, G. Häfner ${ }^{1,7}$, \\ K. Hauschild', M. Heine ${ }^{16}$, C. Henrich ${ }^{5}$, I. Homm ${ }^{5}$, F. Ibrahim', , t. W. Iskra ${ }^{13,15}$, P. Ivanov4, \\ S. Jazrawi ${ }^{3,4}$, A. Korgul ${ }^{9}$, P. Koseoglou ${ }^{5,21}$, T. Kröll ${ }^{5}$, T. Kurtukian-Nieto ${ }^{22}$, L. Le Meur ${ }^{19}$,

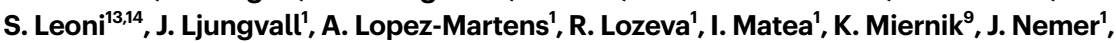 \\ S. Oberstedt ${ }^{23}$, W. Paulsen ${ }^{2}$, M. Piersa ${ }^{9}$, Y. Popovitch', C. Porzio ${ }^{13,14,24}$, L. Qi', D. Ralet ${ }^{25}$, \\ P. H. Regan ${ }^{3,4}$, K. Rezynkina ${ }^{26}$, V. Sánchez-Tembleque ${ }^{12}$, S. Siem ${ }^{2}$, C. Schmitt ${ }^{16}$, \\ P.-A. Söderström ${ }^{5,27}$, C. Sürder ${ }^{5}$, G. Tocabens', V. Vedia ${ }^{12}$, D. Verney', N. Warr ${ }^{7}$, B. Wasilewska ${ }^{15}$, \\ J. Wiederhold ${ }^{5}$, M. Yavahchova ${ }^{28}$, F. Zeiser ${ }^{2}$ \& S. Ziliani ${ }^{13,14}$
}

\begin{abstract}
When a heavy atomic nucleus splits (fission), the resulting fragments are observed to emerge spinning ${ }^{1}$; this phenomenon has been a mystery in nuclear physics for over 40 years ${ }^{2,3}$. The internal generation of six or seven units of angular momentum in each fragment is particularly puzzling for systems that start with zero, or almost zero, spin. There are currently no experimental observations that enable decisive discrimination between the many competing theories for the mechanism that generates the angular momentum ${ }^{4-12}$. Nevertheless, the consensus is that excitation of collective vibrational modes generates the intrinsic spin before the nucleus splits (pre-scission). Here we show that there is no significant correlation between the spins of the fragment partners, which leads us to conclude that angular momentum in fission is actually generated after the nucleus splits (post-scission). We present comprehensive data showing that the average spin is strongly mass-dependent, varying in saw-tooth distributions. We observe no notable dependence of fragment spin on the mass or charge of the partner nucleus, confirming the uncorrelated post-scission nature of the spin mechanism. To explain these observations, we propose that the collective motion of nucleons in the ruptured neck of the fissioning system generates two independent torques, analogous to the snapping of an elastic band. A parameterization based on occupation of angular momentum states according to statistical theory describes the full range of experimental data well. This insight into the role of spin in nuclear fission is not only important for the fundamental understanding and theoretical description of fission, but also has consequences for the $\gamma$-ray heating problem in nuclear reactors ${ }^{13,14}$, for the study of the structure of neutron-rich isotopes ${ }^{15,16}$, and for the synthesis and stability of super-heavy elements ${ }^{17,18}$.
\end{abstract}




\section{Article}

The stability of heavy atomic nuclei is governed by a delicate balance between the Coulomb repulsion of the protons that attempt to deform the nucleus, the nuclear surface tension driving the nucleus towards spherical configurations, and quantum shell effects, which add extra stability for certain nuclear shapes. Fission occurs when there is a perturbation of this balance in favour of the Coulomb repulsion. It is an exothermic, dynamical process that begins as an instability in the nuclear shape, which, after passing the point of no return (the saddle point), becomes more and more elongated. The nascent fragments form a neck as they move rapidly apart, which quickly snaps (scission). Shell effects in the nascent fragments give rise to certain favoured mass splits, which for low-energy fission of actinide nuclei (typically containing about 240 nucleons) produces a light fragment of mass $A \approx 100$ and a heavy fragment of mass $A \approx 140$. After scission, the decay of each excited fragment is a statistical process. It initially proceeds through efficient removal of excitation energy via emission of typically $0-2$ neutrons and $1-3$ high-energy $\gamma$-rays. Subsequently, the emission of several more $\gamma$-rays, which usually carry away two units of angular momentum each, removes the majority of the angular momentum and the remaining excitation energy. This prompt de-excitation process ends at the fragment ground states, usually within a few nanoseconds ${ }^{19}$.

There are many competing theories for how a fissioning nucleus generates its intrinsic angular momentum, and where in the above sequence of events it occurs. One class of explanations proposes that it arises from the excitation of collective vibrational modes such as bending, wriggling, tilting and twisting of the system before it splits (pre-scission). These theories suggest that the vibrations are either initiated by thermal excitations ${ }^{4-6}$, arise from quantum fluctuations ${ }^{7,8}$, or both ${ }^{9}$. Post-scission theories suggest that the angular momenta are generated either from Coulomb forces ${ }^{10}$ or from deformed fragments that have coupled orientations ${ }^{11,12}$. Since the angular momentum is quickly carried away by the $\gamma$-rays, the experimental study of the generation mechanism necessarily involves detailed observation of the prompt $\gamma$-ray emission.

Experimental attempts to understand the intrinsic spin generation started with low-resolution detection of prompt fission $\gamma$-rays correlated with the indirect detection (plastic scintillator ${ }^{20}$ and surface barrier detectors $^{21}$ ) of fragment mass, which revealed saw-tooth shapes in the $\gamma$-ray yields that are strongly related to spin. The major difficulty was the separation of $\gamma$-rays emitted from the two fragments, and the existence of these patterns was called into question in a later experiment where no saw-tooth pattern was observed ${ }^{22}$. Another experimental approach involves spectroscopy of isomeric (long-lived) excited states found in certain nuclei. Measurements of isomer population are highly sensitive to small relative changes in spin. However, only a small subset of all the isotopes produced in fission have such isomeric states and it is difficult to measure trends over a large range in mass ${ }^{23}$. In this work, we use a third technique ${ }^{24}$ based on high-resolution spectroscopy, which allows both separation of $\gamma$-rays from the two fragments and the study of trends over large mass ranges.

To probe intrinsic generation of angular momentum also requires systems with initial spin of zero or almost zero, namely spontaneous fission or neutron-induced fission. Heavy-ion or charged-particle-induced fission reactions are unsuitable because they generate high initial angular momenta ${ }^{25}$, which can obscure the origin of the intrinsic spin.

We present here unique and extensive experimental data obtained from fission experiments carried out at the ALTO facility of the IJC Laboratory in Orsay, France, with the LICORNE directional neutron source ${ }^{26,27}$ coupled to the high-performance $v$-Ball $\gamma$-ray spectrometer ${ }^{28}$. We carried out high-resolution spectroscopy of fast-neutron-induced fission of ${ }^{232} \mathrm{Th}$ and ${ }^{238} \mathrm{U}$, and the spontaneous fission of ${ }^{252} \mathrm{Cf}$ with the addition of an ionization chamber ${ }^{29}$.

\section{Results}

For each of the three systems studied we identified characteristic $\gamma$-ray decay patterns of excited states in around 30 even-even nuclei (with

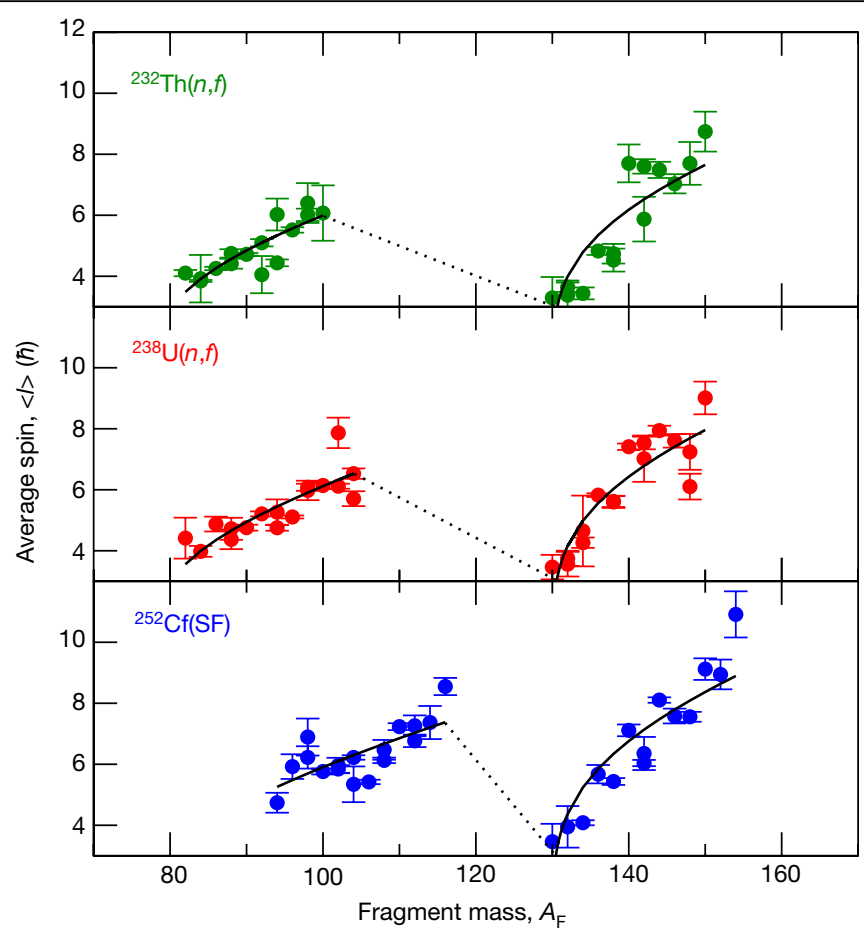

Fig. 1 | Dependence of average spin on fragment mass. Average spins extracted for even-even nuclei produced in fast-neutron-induced fission of ${ }^{232} \mathrm{Th},{ }^{238} \mathrm{U}$ and the spontaneous fission of ${ }^{252} \mathrm{Cf}$ are presented along with statistical uncertainties (error bars represent \pm 1 standard deviation, s.d.). Single-parameter fits to the data are shown in black lines. The fitting parameterization developed to explain the mechanism that generates angular momentum is presented in the section 'Discussion'.

even numbers of both protons and neutrons). For each even-even fission fragment we extracted the average spin after neutron emission using a method developed at the University of Manchester ${ }^{24}$, which combines all the available $\gamma$-ray transition intensity and coincidence information (see Methods).

Our results (Fig. 1) definitively confirm that fragment spins vary strongly as a function of fragment mass in saw-tooth distributions, similar to the patterns previously observed in $\gamma$-ray yields ${ }^{20,21}$. We note that a given fragment spin appears to depend only on the fragment mass, with no observable relationship to the mass of the system that emits it nor to the mass or charge of the partner nucleus with which it emerged. This observation does therefore not support theoretical explanations based on post-scission Coulomb effects ${ }^{10}$, where a dependence of spin on the product of the fragment charges, $Z_{1} Z_{2}$, would be expected.

Additionally, large asymmetries in average spin are observed for certain fragment pair combinations (for example, ${ }^{86} \mathrm{Se}$ and ${ }^{150} \mathrm{Ce}$ from ${ }^{238} \mathrm{U}(n, f)$ ), where the spin of the heavy fragment can be more than double that of its light partner. The existence of such asymmetries does not support the post-scission explanation based on coupled orientations of deformed fragments ${ }^{11,12}$, which explicitly predicts spins of equal magnitudes. Indeed, the existence of such large spin asymmetries provokes the question of how spin generation could possibly occur pre-scission if the fragments are in contact and participating in a correlated collective motion. In that case, expected fragment spins at scission would be $+I$ and $-I$ units. To investigate further, we studied the correlation between spins of the most strongly populated fragments in the ${ }^{238} \mathrm{U}(n, f)$ reaction. For a given nucleus, $\gamma$-ray transitions of increasing spin were selected from its partner nucleus, constraining the partner population to higher and higher spins. We then examined how the average spin of the given nucleus evolved in response (Fig. 2). For example, the most strongly populated partner nucleus of ${ }^{96} \mathrm{Sr}$ is ${ }^{140} \mathrm{Xe}$. 


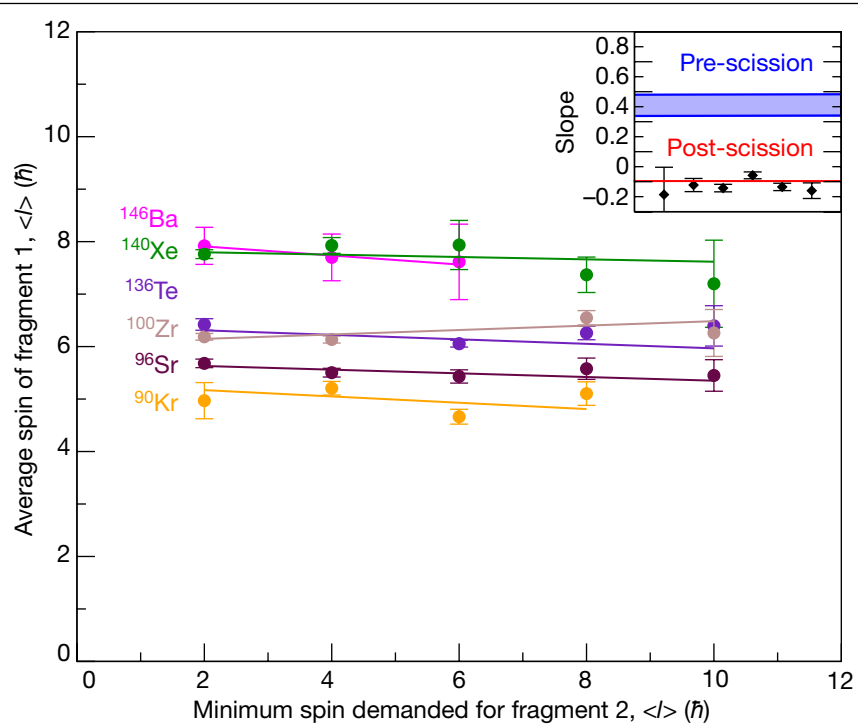

Fig. 2 Correlation between fragment spins. Correlations between fragment and partner spins for the six most strongly populated fragments in the ${ }^{238} \mathrm{U}(n, f)$ reaction with associated statistical uncertainties (error bars represent \pm 1 s.d.). Weighted linear fits to the data points for each nucleus are shown. The fitted slopes are compared to the expected slopes for the spin mechanisms pre-scission with correlated spins ('Pre-scission') or post-scission with uncorrelated spins ('Post-scission') in the inset. The blue band ('Pre-scission') was determined from Monte-Carlo simulations of the de-correlating effects of the neutrons and statistical $\gamma$-rays (see Methods and Extended Data Fig. 3).

By demanding observation of a $\gamma$-ray emitted from the lowest $8^{+}$state in ${ }^{140} \mathrm{Xe}$ we constrain this nucleus to be populated with average spins of higher than 8 units of angular momentum. The corresponding average spin in ${ }^{96} \mathrm{~S} r$ is deduced by measuring the corresponding coincident $\gamma$-ray intensities. By varying the spin conditions and the isotopes studied, we obtain the fragment spin correlations.

The observed slopes are clearly consistent with zero, suggesting an uncorrelated, post-scission spin-generation mechanism. The overall slope from the combined data is within a $2 \sigma$ confidence interval of $[-0.04,0.01]$, compatible with no significant correlation between fragment spins and incompatible with correlated pre-scission spin generation. The data do not support pre-scission theoretical explanations ${ }^{4-9}$, confirming what was suspected from the large spin asymmetries (Fig. 1). It appears that each fragment has no 'knowledge' of the spin generated in its partner.

This unexpected conclusion may resolve the historical controversy surrounding previous experimental results $\mathrm{s}^{20-22}$. For fragment spins that are generated independently, the event-by-event correlations measured in ref. ${ }^{22}$ would not be expected to generate a saw-tooth pattern in the $\gamma$-ray yield measurements. Hence, this absence of the saw-tooth pattern may support rather than contradict our current findings.

\section{Discussion}

A post-scission, uncorrelated origin of angular momentum suggests that the fragments have become two separate, independent quantal systems. This can be viewed from both macroscopic and statistical/ single-particle points of view.

\section{Post-scission generation of two independent torques}

Macroscopically, we suggest that fragments acquire their spin in a process analogous to the snapping of an elastic band. A neck forms between the two emerging fragments, which undergoes first a stretching, then a rupture and finally a relaxation during which the potential energy
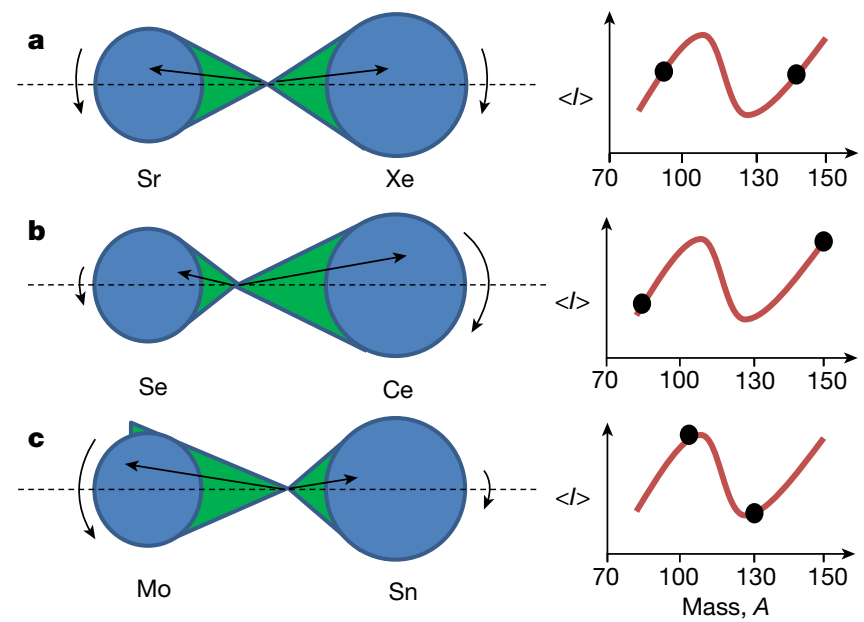

Fig. 3 | Schematic diagram of post-scission angular momentum generation. Independent torques for different scission configurations are shown, with neck nucleons displayed in green. The straight black arrows illustrate sizes and example directions of the linear momentum vectors that generate the associated angular momenta. The corresponding positions on the saw-tooth distribution of the resulting average spins are shown on the right.

from the deformed neck (analogous to stretched elastic) transforms into kinetic energy. For asymmetric fission of the actinide nuclei we assume a double cluster, with the cores of the nascent fragments lying near doubly closed shells and the remaining nucleons from the neck shared between them after rupture (Fig. 3).

We suggest that the nucleons from both halves of the ruptured neck drive the generation of angular momentum in each fragment. The relative sizes of torques will depend on the number of neck nucleons and thus the precise location of the neck rupture, that is, the configuration at scission. Classically, the neck would rupture in the middle at its weakest point. However, in the subatomic world a gap can appear at any point ${ }^{30}$, with decreasing probabilities for more extreme partitions. We suggest that how the system arrives at a specific scission configuration will not have any subsequent impact on the generation of post-scission spin and that the fragments retain no 'memory' of their formation after scission.

At scission, the former neck nucleons are located far from the centres of mass of the newly born fragments in two very elongated configurations. Such extreme elongations have large surface energies, which provide the restoring forces towards more spherical shapes. Fluctuations in the aggregate direction of motion of these former neck nucleons generate the two independent torques. Small angular deviations from the fission axis of the collective nucleon motion must occur, owing to Heisenberg's uncertainty principle for spin/orientation of a system ${ }^{31}$. Uncertainties in the direction of the resulting linear momentum along the fission axis will result in small perpendicular components that will generate a distribution of angular momenta. Angular momenta in both fragments will point in a plane perpendicular to the fission axis, consistent with previous experiments ${ }^{1}$, although there will be no correlation or constraint on their relative orientations. The resulting orbital angular momentum, $\mathbf{I}_{\mathrm{o}}$, of the fragments with respect to each other, generated by the components of the motion perpendicular to the fission axis, ensures the conservation of the total angular momentum: $\mathbf{I}_{1}+\mathbf{I}_{2}+\mathbf{I}_{0}=\mathbf{0}$.

The dramatic fragment shape-change from elongated to more spherical shapes will also generate heat as the surface energy converts into internal excitation energy, setting the stage for subsequent evaporation of neutrons. Angular momentum, excitation energy and emitted neutron multiplicity will thus be strongly correlated with each other. 


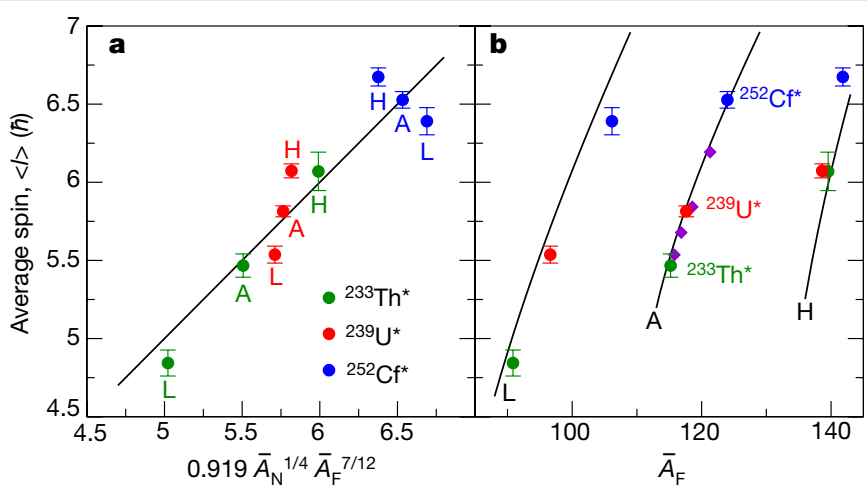

Fig. 4 | Relationship between average fragment spins and average masses. Fragment yield-averaged data with statistical uncertainties (error bars represent \pm 1 s.d.) are shown. a, Data are compared to predictions from the parameterization for the three fissioning systems, with light peak $(\mathrm{L})$, heavy peak $(\mathrm{H})$ and average for the system (A) marked. The superscripts are the exponents in the fitting function of equation (2); see also Extended Data Fig. 4.b, The same data are plotted as a function of the average fragment mass $\bar{A}$ with the black lines showing predictions for other systems from the parameterization. Purple diamonds show specific predictions for the major fissile isotopes, ${ }^{233,235} \mathrm{U},{ }^{239} \mathrm{Pu}$ and in addition,

${ }^{245} \mathrm{Cm}$. The asterisks conventionally denote that the nucleus is a compound nuclear system, thus indicating the absorption of a neutron in the cases of ${ }^{238} \mathrm{U}+n={ }^{239} \mathrm{U}^{*}$ and ${ }^{232} \mathrm{Th}+n={ }^{233} \mathrm{Th}^{*}$. However, ${ }^{252} \mathrm{Cf}^{*}$ has not absorbed a neutron since it undergoes spontaneous fission.

Indeed, similar saw-tooth distributions are known to occur in average neutron multiplicities as a function of fragment mass ${ }^{32,33}$.

\section{Comparison of the variation in average spins to that expected from statistical theory}

In the statistical/single-particle view, if the newly formed fragments are independent, then their excited states would be expected to have an angular momentum occupation according to statistical theory. For an excited nucleus, the probability distribution, $P(I)$, of angular momenta, $I$, was first derived by Hans Bethe ${ }^{34}$ and is expected to be:

$$
P\left(I \mid \sigma^{2}\right)=\frac{2 I+1}{2 \sigma^{2}} \exp \left(-\frac{(I+1 / 2)^{2}}{2 \sigma^{2}}\right)
$$

where $\sigma$ is known as a spin-cutoff parameter describing the width of this distribution and is directly related to the average spin value, $I \approx 1.15 \sigma$. From statistical theory (see Methods) we derive a smooth parameterization that can be tested for compatibility with our average spin data (Fig. 1):

$$
I=c A_{\mathrm{N}}^{1 / 4} A_{\mathrm{F}}^{7 / 12}
$$

where $c$ is a constant and the only free fitting parameter, $A_{\mathrm{F}}$ is the fragment mass, and $A_{\mathrm{N}}$ is the mass of neck nucleons. For light and heavy fragments we use values of $A_{\mathrm{N}}=\left(A_{\mathrm{F}}-78\right)$ and $A_{\mathrm{N}}=\left(A_{\mathrm{F}}-130\right)$ near the doubly magic $\mathrm{Ni}$ and $\mathrm{Sn}$ shell closures. The derivation presented here has limitations and is not intended as a full description, but as an illustration of the idea (see Methods). An extended theoretical description would involve more complex dependencies of the parameters owing to structure effects.

Six independent fits using the above parameterization for each light and heavy peak in the three different systems were performed. The fitted constants are remarkably similar, with a mean of $c=0.196$ and standard deviation of $\sigma_{\mathrm{c}}=0.009$, a relative variation of only about $4 \%$, suggesting that the fragment spins fall on a universal curve. This simple parameterization thus appears to capture the main ingredients of the spin-mass relationship. We conclude that the experimentally observed variation in the average spins is thus consistent with what is expected from statistical theory for a post-scission, uncorrelated, spin-generation mechanism. There may be other second-order effects (for example, Coulomb forces) that are not yet accounted for, but these are clearly small.

A concise suggestion for the mechanism that generates the intrinsic angular momentum in the light of our data is as follows, although we recognize that other interpretations may also be possible. A fissioning nucleus that starts with zero or near-zero spin undergoes: (i) unstoppable shape instability from Coulomb forces; (ii) formation of a neck between the two emerging fragment clusters; (iii) neck stretching and rupture (scission) with the birth of two deformed, newly independent quantal systems; (iv) shape relaxation of each fragment as the surface potential energy converts to excitation of the internal nucleonic degrees of freedom; (v) the resulting occupation of different angular momentum states occurring in accordance with statistical theory for two independent excited nuclei. In the equivalent macroscopic picture, the last two steps can also be seen as: (iv) a shape relaxation where aggregate collective motions of the nucleons have off-axis components generating two independent torques; (v) the statistical distributions of torques creates two independent distributions of spin.

\section{Consequences}

Understanding the angular momentum generated in fission is important for fundamental reasons, but also has consequences for other fields. In nuclear energy applications, fragment spin is related to reactor $\gamma$-ray heating effects ${ }^{13,14}$, either through the number of prompt $\gamma$-rays that transport it during reactor operation, or the delayed $\gamma$-rays from isomeric states that contribute to the decay heat after reactor shutdown. For these reasons, many recent measurements of prompt $\gamma$-ray characteristics, such as the average multiplicity $M_{\gamma}$, have been carried out $^{35-37}$. At present, only purely empirical connections between these characteristics and the mass of the fissioning system have been made ${ }^{38}$. However, we are now able to understand better the underlying fundamental relationships if we combine our data with known fragment yield information (Fig. 4). Here, we manage to relate two independent average quantities, the mass and the spin, for light, heavy and average fragment masses, and use our parameterization to make predictions for other systems.

We also note that fission is a production mechanism used to study the structure of exotic nuclei ${ }^{15,16}$. Thus, understanding spin generation will allow determination of which excited nuclear states can be accessed. Finally, outside the actinide region of the periodic table, fragment-yield distributions evolve as a result of the changing influence of shell closures. For example, a transition from asymmetric to symmetric fission occurs for nuclei beyond ${ }^{258} \mathrm{Fm}\left(\right.$ ref. $\left.^{39}\right)$. In the recently discovered region of $\beta$-delayed fission ${ }^{40}$ around ${ }^{180} \mathrm{Hg}$, the shell effects that drive the configuration at scission are not well understood. For fission regions that are less well explored, measurements of spin-sensitive $\gamma$-ray data could yield valuable information on neck formation and the relevant shell closures involved.

\section{Conclusion}

A full theoretical description of nuclear fission requires the incorporation of the mechanism that generates intrinsic angular momentum. We have presented extensive experimental data on fragment spins in different systems from which it is now finally possible to discriminate between the many competing theoretical explanations of this mechanism. We show that fragment spins are uncorrelated, revealing the post-scission nature of the mechanism. Theoretical explanations based on pre-scission collective vibrations ${ }^{4-8}$, post-scission Coulomb excitations ${ }^{10}$ or coupling through fragment deformations are not supported by our data ${ }^{9,11}$. A parameterization based on the expected occupation of 
spin states according to statistical theory describes the experimentally observed mass dependence of average spins well.

\section{Online content}

Any methods, additional references, Nature Research reporting summaries, source data, extended data, supplementary information, acknowledgements, peer review information; details of author contributions and competing interests; and statements of data and code availability are available at https://doi.org/10.1038/s41586-021-03304-w.

1. Wilhelmy, J. B., Cheifetz, E., Zared, R. C., Thompson, S. G. \& Bowman, H. R. Angular momentum of primary products formed in the spontaneous fission of ${ }^{252} \mathrm{Cf}$. Phys. Rev. C 5, 2041 (1972).

2. Schmidt, K.-H. \& Jurado, B. Review on the progress in nuclear fission-experimental methods and theoretical descriptions. Rep. Prog. Phys. 81, 10630 (2018).

3. Andreyev, A. N., Nishio, K. \& Schmidt, K.-H. Nuclear fission: a review of experimental advances and phenomenology. Rep. Prog. Phys. 81, 016301 (2018).

4. Rasmussen, J. O., Nörenberg, W. \& Mang, H. J. A model for calculating the angular momentum distribution of fission fragments. Nucl. Phys. A 136, 465 (1969).

5. Moretto, L. G. \& Peaslee, G. F. \& Wozniak, G. J. Angular-momentum-bearing modes in fission. Nucl. Phys. A 502, 453 (1989).

6. Mișicu, S., Săndulescu, A., Ter-Akopian, G. M. \& Greiner, W. Angular momenta of even-even fragments in the neutronless fission of ${ }^{252} \mathrm{Cf}$. Phys. Rev. C 60, 034613 (1999).

7. Shneidman, T. M. et al. Role of bending mode in generation of angular momentum of fission fragments. Phys. Rev. C 65, 064302 (2002).

8. Gönnenwein, F., Tsekhanovich, I. \& Rubchenya, V. Angular momentum of near-spherical fission fragments. Int. J. Mod. Phys. E 16, 410-424 (2007).

9. Bonneau, L., Quentin, P. \& Mikhailov, A. N. Scission configurations and their implication in fission-fragment angular momenta. Phys. Rev. C 75, 064313 (2007).

10. Hoffman, M. M. Directional correlation of fission fragments and prompt gamma rays associated with thermal neutron fission. Phys. Rev. 133, B714 (1964).

11. Mikhailov, I. N. \& Quentin, P. On the spin of fission fragments, an orientation pumping mechanism. Phys. Lett. B 462, 7 (1999).

12. Bertsch, G. F., Kawano, T. \& Robledo, L. M. Angular momentum of fission fragments. Phys. Rev. C 99, 034603 (2019).

13. Rimpault, G. et al. Needs of accurate prompt and delayed $y$-spectrum and multiplicity for nuclear reactor designs. Phys. Proc. 31, 3-12 (2012)

14. Lemaire, M., Vaglio-Guadard, C., Lyoussi, A. \& Reynard-Carette, C. For a better estimation of gamma heating in nuclear material-testing reactors and associated devices: status and work plan from calculation methods to nuclear data. J. Nucl. Sci. Technol. 52, 9 (2015)

15. Dudouet, J. et al. ${ }^{96} \mathrm{Kr}$-low- $Z$ boundary of the island of deformation at $N=60$. Phys. Rev. Lett. 118, 162501 (2017)

16. Ha, J. et al. Shape evolution of neutron-rich ${ }^{106,108,110} \mathrm{Mo}$ isotopes in the triaxial degree of freedom. Phys. Rev. C 101, 044311 (2020).

17. Zagrebaev, V. I., Aritomo, Y., Itkis, M. G., Oganessian, Yu. Ts. \& Ohta, M. Synthesis of superheavy nuclei: how accurately can we describe it and calculate the cross sections? Phys. Rev. C 65, 014607 (2001)
18. Itkis, M. G., Vardaci, E., Itkis, I. M., Knyazhev, G. N. \& Kozulin, E. M. Fusion and fission of heavy and superheavy nuclei (experiment). Nucl. Phys. A 944, 204-237 (2015)

19. Talou, P. et al. Late-time emission of prompt fission y rays. Phys. Rev. C 94, 064613 (2016).

20. Armbruster, P., Labus, H. \& Reichelt, K. Investigation on the primary spins of the ${ }^{235} \mathrm{U}$ fission fragments. Z. Naturforsch. 26a, 512-522 (1971).

21. Pleasonton, F. Prompt $y$-rays emitted in the thermal-neutron induced fission of ${ }^{233} \mathrm{U}$ and ${ }^{239}$ Pu. Nucl. Phys. A 213, 413-425 (1973).

22. Glässel, P., Schmid-Fabian, R. \& Schwalm, D. ${ }^{252} \mathrm{Cf}$ fission revisited-new insights into the fission process. Nucl. Phys. A 502, 315-324 (1989).

23. Naik, H., Dange, S. P. \& Singh, R. J. Angular momentum of fission fragments in low energy fission of actinides. Phys. Rev. C 71, 014304 (2005).

24. Abdelrahman, Y. et al. Average spins of primary fission fragments. Phys. Lett. B 199, 504-508 (1987)

25. Boutoux, G. et al. Study of the surrogate-reaction method applied to neutron-induced capture cross sections. Phys. Lett. B 712, 319-325 (2012)

26. Lebois, M. et al. Development of a kinematically focused neutron source with the $\mathrm{p}\left({ }^{7} \mathrm{Li}, \mathrm{n}\right)^{7} \mathrm{Be}$ inverse reaction. Nucl. Instrum. Meth. Phys. Res. A 735, 145-151 (2014)

27. Wilson, J. N. et al. The LICORNE neutron source and measurements of prompt $\mathrm{y}$-rays emitted in fission. Phys. Proc. 64, 107-113 (2015)

28. Lebois, M. et al. The v-ball spectrometer. Nucl. Instrum. Meth. Phys. Res. A 960, 163580 (2020)

29. Gaudefroy, L. et al. Impact of Coriolis mixing on a two-quasi-neutron isomer in ${ }^{164} \mathrm{Gd}$ and other $N=100$ isotones. Phys. Rev. C 97, 064317 (2018).

30. Brosa, U., Grossmann, S. \& Müller, A. Nuclear scission. Phys. Rep. 197, 167-262 (1990).

31. Franke-Arnold, S., Barnett, S. M., Leach, J., Courtial, J. \& Padgett, M. Uncertainty principle for angular position and angular momentum. New J. Phys. 6, 103 (2004).

32. Terrell, J. Neutron yields from individual fission fragments. Phys. Rev. 128, 2925 (1962).

33. Göök, A., Hambsch, F. \& Vidali, M. Prompt neutron multiplicity in correlation with fragments from spontaneous fission of ${ }^{252} \mathrm{Cf}$. Phys. Rev. C 90, 064611 (2014).

34. Bethe, H. A. An attempt to calculate the number of energy levels of a heavy nucleus. Phys. Rev. 50, 332 (1936).

35. Oberstedt, A. et al. Improved values for the characteristics of prompt-fission y-ray spectra from the reaction ${ }^{235} \mathrm{U}\left(\mathrm{n}_{\mathrm{th}}, \mathrm{f}\right)$. Phys. Rev. C 87, 051602(R) (2013).

36. Chyzh, A. et al. Systematics of prompt y-ray emission in fission. Phys. Rev. C 87, 034620 (2013).

37. Qi, L. et al. Statistical study of the prompt-fission $y$-ray spectrum for ${ }^{238} U(n, f)$ in the fast-neutron region. Phys. Rev. C 98, 014612 (2018).

38. Valentine, T. E. Evaluation Of Prompt Fission Gamma Rays For Use In Simulating Nuclear Safeguard Measurements. Oak Ridge National Laboratory Report TM-1999/300, https:// www.osti.gov/servlets/purl/753485-4TSmOw/webviewable/ (US Department of Energy Office of Scientific and Technical Information, 2001).

39. Flynn, K. F. et al. Distribution of mass in the spontaneous fission of ${ }^{256} \mathrm{Fm}$. Phys. Rev. C 5, 1725 (1972).

40. Andreyev, A. et al. New type of asymmetric fission in proton-rich nuclei. Phys. Rev. Lett. 105,252502 (2010).

Publisher's note Springer Nature remains neutral with regard to jurisdictional claims in published maps and institutional affiliations.

(c) The Author(s), under exclusive licence to Springer Nature Limited 2021 


\section{Methods}

\section{Experimental setup}

Samples of ${ }^{238} \mathrm{U}(81 \mathrm{~g})$ and ${ }^{232} \mathrm{Th}(129 \mathrm{~g})$ were irradiated with a pulsed neutron beam from the LICORNE neutron source (400-ns period) in the centre of the $\mathrm{v}$-Ball spectrometer for total acquisition times of $216 \mathrm{~h}$ and $450 \mathrm{~h}$, respectively. The average neutron energy that provoked fission was $1.9 \mathrm{MeV}$. Triggerless data from the 184 detectors in the $\mathrm{v}$-Ball array were written to disk at high data rates of typically 1-3 million $\gamma$-ray hits per second, and processed later offline. Each detected $\gamma$-ray energy was associated with a unique 64-bit time stamp accurate to sub-nanosecond precision, thanks to the state-of-the-art FASTER digitization system ${ }^{41} \cdot \gamma$-ray coincidence events were identified offline with a minimum trigger condition defined as at least two unsuppressed high-resolution germanium $(\mathrm{Ge})$ detectors and at least one other detector module (bismuth germinate (BGO) or $\mathrm{LaBr}_{3}$ ) firing within a short 80-ns time window. These events were subsequently sorted into twoand three-dimensional histograms for further offline analysis. An additional dataset was gathered from the ${ }^{252} \mathrm{Cf}$ spontaneous fission source inside an ionization chamber ${ }^{29}$ placed in the centre of the $\mathrm{v}$-Ball array for $52 \mathrm{~h}$. With this latter setup, one fragment was detected in-flight, while the other fragment was stopped in the backing of the sample.

\section{Data analysis}

Examples of $\gamma$-ray coincidence spectra are shown in Extended Data Fig. 1 and Extended Data Fig. 2, which support the main findings of the Article. The lack of dependence of the ${ }^{140} \mathrm{Xe}$ intensity pattern on the fissioning system is shown in Extended Data Fig. 1, and the lack of correlation between ${ }^{140} \mathrm{Xe}$ and ${ }^{96} \mathrm{Sr}$ fragment intensity patterns is shown in Extended Data Fig. 2.

The main experimental data on average spins after neutron emission presented in this Article rely on a method initially developed at Manchester University in the late 1980s and described fully in ref. ${ }^{24}$. It will henceforth be referred to as the Manchester Spin Method (MSM).

The MSM relies on measuring the relative intensity of every resolvable $\gamma$-ray transition for a given nucleus populated in the reaction of interest. At each level with spin $I$, the intensity difference between the observed ingoing and outgoing transitions is computed. This difference is defined as the direct side-feeding, $S$, of the state. The average spin populated is therefore the side-feeding-weighted average of the level spins over all $n$ levels, $\langle I\rangle=\sum_{i=1 . . n} I_{i} S_{i} / \sum_{i=1 . . n} S_{i}$. A further small correction in the result is necessary to account for the angular momentum carried away by the statistical transitions, which depends on the reaction and is deduced from $\gamma$-ray decay models at around one extra unit in the case of fission.

The MSM condenses all the available $\gamma$-ray intensity and coincidence data for a given nucleus into a single number: the average spin after neutron emission. It is thus a powerful experimental tool with which to study angular momentum effects in nuclear reactions. The method measures a cumulative intensity flow through many different excited states, all of which will eventually reach the ground state. There is a redundancy in the measured information and the method has a low sensitivity to individual $\gamma$-ray intensities (that is, a large perturbation in the intensity value of any particular $\gamma$ ray from the decay pattern has a small impact on the result). For example, the inclusion or exclusion of the intensities of transitions from states other than those with the lowest energy for a given spin (so-called yrast states) levels in the calculation is seen to have very little impact on the result (see Methods section 'Non-inclusion of weaker transitions'). If the yrast sequence of transitions is observed, then an average spin can be extracted from the data.

We note that the first experiment where the MSM was applied used only 12 Compton suppressed small-volume Ge detectors to study heavy-ion induced fission. In this work, we measure neutron-induced fission with a high-performance third-generation $\gamma$-ray spectrometer with 106 large-volume, Compton-suppressed Ge crystals (that is, $\gamma$-rays that scatter out of the Ge crystal and into the surrounding BGO scintillator are vetoed) and state-of-the-art, triggerless signal digitization technology.

\section{Application of the MSM to v-Ball data}

We measured the average spin in around 30 even-even nuclei in each system (see Extended Data Tables 1, 2 and 3). Even-even nuclei have relatively simple, well known decay schemes and are generally much easier to study. Even-odd and odd-odd isotopes often have highly fragmented decay patterns with many low-energy transitions, which are difficult to detect. The presence of a neutron beam pulsation, or-as for the case of the spontaneously fissioning ${ }^{252} \mathrm{Cf}(\mathrm{SF})$-direct detection of one of the fission fragments in the ionization chamber, is crucial for distinguishing between $\gamma$ rays from prompt fission and those from subsequent fragment $\beta$-decays. This latter source of $\gamma$ rays is usually associated with low multiplicity events $\left(M_{\gamma} \approx 2-3\right)$, but for certain isotopes can be comparable to that of fission. Emission of $\gamma$-rays after $\beta$-decays is uncorrelated in time, whereas $95 \%$ of prompt fission $\gamma$-rays are emitted within a few nanoseconds of the beam pulse or fission event. Without the beam pulsation, $\gamma$-rays from $\beta$-decay and prompt fission events are difficult to discriminate. This can lead to difficulties in extracting fragment average spin from intensity measurements, since population of nuclei via both processes occurs. The closer to stability the nucleus, the more of a problem this represents. ${ }^{252} \mathrm{Cf}(\mathrm{SF})$ has been extensively studied with spectroscopy over the last twenty years ${ }^{42,43}$ but mostly from datasets without direct fission fragment detection, where the primary focus has been on extending knowledge on the nuclear structure of exotic neutron-rich nuclei. Spin effects in ${ }^{252} \mathrm{Cf}(\mathrm{SF})$ may have been difficult to study without an ability to discriminate $\gamma$-rays from fission and $\beta$-decay.

\section{$\boldsymbol{\gamma}$-ray coincidence data and efficiency calibrations}

Application of the MSM requires determination of the $\gamma$-ray full energy peak detection efficiency over a wide range of energy: $100 \mathrm{keV}$ to $5 \mathrm{MeV}$. Each of the three systems studied $\left({ }^{232} \mathrm{Th},{ }^{238} \mathrm{U},{ }^{252} \mathrm{Cf}\right)$ has its own unique efficiency curve owing to different target/chamber geometries producing slightly different self-shielding effects at lower energies. These were determined by combining GEANT IV ${ }^{44}$ simulations of the setup for the highest-energy part (2-5 MeV), source measurements, and measurements from the fission coincidence data for the lowest-energy part (100-500 keV). For the lowest part of the energy range, self-shielding effects in the massive ${ }^{232} \mathrm{Th}$ and ${ }^{238} \mathrm{U}$ targets are particularly important, and are difficult to simulate owing to the complex, non-uniform distribution of fissions within these targets. The drop in efficiency below $200 \mathrm{keV}$ is substantial and is measured from the experimental data by gating on $\gamma$-ray yrast cascades in rotational nuclei (for example, Ce and Mo) from above and measuring efficiency ratios for the transitions below. Uncertainties on these efficiencies are included in the data analysis for the measurement of $\gamma$-ray intensities and in the subsequent deduction of average spins after neutron emission.

\section{Fitting procedures}

Global fits of many thousands of $\gamma$-ray coincidences were performed in two dimensional (2D) $\gamma-\gamma$ coincidence matrices using the Radware software package ${ }^{45}$. Two dimensional analysis is essential to measure 4+ state side-feedings. Since many nuclei share similar transition energies, a global 2D fitting procedure is needed for accurate measurements of transition intensities. Odd-even and odd-odd nuclei also need to be included so that all the possible coincidences can be identified in a particular matrix slice or region. Level scheme information from the evaluated ENSDF libraries ${ }^{46}$ containing level spins, excitation energies, transition energies and coincidence relationships are used as the starting point for each nucleus. Peak width parameters are fixed from a pre-determined width calibration as a function of energy. 
The intensities, $G_{k}$, and energies of the observable transitions are then fitted simultaneously for all nuclei in a global fit with thousands of free parameters. Subsequent local fits for each nucleus are then performed to check convergence at the local level, with global parameters fixed and only local parameters free to vary. Global and local fits are then repeated iteratively until convergence is achieved. At each stage, Radware calculates a $\chi^{2} /$ degrees of freedom, which is used to verify and assure convergence for each nucleus. Additionally, Radware also allows for powerful visual comparisons between the fitted $\gamma$-ray coincidences and the experimental spectral data. This facilitates a large number of visual checks to ensure the level scheme of each fragment is correctly fitted and the local fit has fully converged. Global fits serve only as second-order corrections to fit properly the rare occasions when one fragment contains a pair of transitions of similar energies to those in another fragment.

To process the results of the fitting procedure and to extract side-feedings and average spins for each nucleus, new software has been developed that operates on the fitted intensity and peak position output from Radware. The side-feeding $S_{i}$ of each level is computed from the sum of all observable transition intensities, $G_{k}$, feeding in and out of each level.

The software checks the level scheme transition intensities for self-consistency. Negative side-feedings are unphysical and if detected may signal a potential problem with the fitting of transitions feeding in or out of a particular level. Finally, the code computes the average spin for each nucleus studied by combining the level spins $I_{i}$ and the side-feeding $S_{i}$ information $\langle I\rangle=\sum_{i=1 . . n} I_{i} S_{i} / \sum_{i=1 . . n} S_{i}$.

\section{Propagation of uncertainties and variance-covariance}

The computed statistical uncertainty on the intensity of a particular transition is dependent on statistical variations in the number of counts at coincident peak positions in two dimensions. A relative uncertainty in the level of background of $5 \%$ is assumed along with a typical relative uncertainty in the detection efficiency of 3\%. For transitions below $200 \mathrm{keV}$ the relative uncertainty on the detection efficiency rises to $20 \%$ owing to the substantial drop in detection efficiency over this energy range.

To determine the uncertainties on the extracted average spins $\langle 1\rangle$, the uncertainties on the fitted experimental intensity data are propagated through the MSM. However, the intensities, $G_{k}$, and side-feedings, $S_{i}$, are not necessarily independent and correlations may exist between these parameters. Therefore, correct mathematical treatment of error propagation requires the incorporation of potential correlated sources of uncertainty. Analysis of variances and covariances are needed first for the intensities, $G_{k}$, to determine the uncertainties on the $S_{i}$ and then for the side-feedings to determine the error on average spin $\sigma_{\langle I\rangle}$ in the following way:

$$
\sigma_{\langle l\rangle}^{2}=\sum_{i=1 . . n} I_{i}^{2} \sigma^{2}\left(S_{i}\right)+\sum_{i=1 . . n} \sum_{j=1 . . n} I_{(i \neq j)}^{2} I_{j}^{2} \operatorname{cov}\left(S_{i}, S_{j}\right)
$$

where $\operatorname{cov}\left(S_{i}, S_{j}\right)$ is the matrix of covariances.

Given that the v-Ball detector array uses Ge detectors with excellent resolution, a high detector granularity, and a 'low' overall efficiency $(\sim 5 \%)$, the vast majority of covariances between intensity parameters are zero. Within the same level scheme, the off-diagonal elements of the variance-covariance matrix are typically $(<0.05)$, so the independence of the $G_{k}$ values can be considered a realistic assumption. However, the same cannot be said of the $S_{\mathrm{i}}$ values, which are computed from intensity differences between neighbouring transitions in the scheme. The adjacent side-feedings $S_{\mathrm{i}}$ are thus strongly correlated with each other, giving rise to both large negative and positive off-diagonal elements in the corresponding covariance matrix (about $|0.4-0.8|$ ). To perform the propagation requires the computation of a covariance matrix $\operatorname{cov}\left(S_{i}, S_{j}\right)$ for each data point of $\langle l\rangle$. This is complex and laborious, and given the number of data points, each one derived from a separate level scheme and having its own unique set of coincidence relationships, this procedure for uncertainty calculation is challenging.

A more practical method for obtaining good estimates of the statistical errors associated with each average $<1>$ is to fit the side-feeding distribution as in Extended Data Fig. 4 and use the resulting uncertainty on the fitted average of this distribution. Here, there may be some small dependence of the uncertainty on the exact form of the fitting function chosen. This procedure for uncertainty estimation yields uncertainties comparable in size to the application of variance-covariance analysis.

Using the example of the ${ }^{238} \mathrm{U}(n, f)$ coincidence data, the observable intensities vary from the strong, for example, ${ }^{140} \mathrm{Xe} 4^{+} \rightarrow 2^{+}$at $3.42(11) \%$ of the total yield, to the very weak $14^{+} \rightarrow 12^{+}$in ${ }^{150} \mathrm{Ce}$ at $0.024(16) \%$ of the total yield. The median relative statistical uncertainties on transition intensities from the global fit is $13 \%$, and for level side-feedings is $24 \%$. This gives rise to a typical relative average spin uncertainty of around $5 \%$.

\section{Sensitivity of the MSM}

The level of accuracy, or sensitivity, of the MSM is an important question. To what extent are side-feeding distributions measured at or near the yrast line distorted by local quirks of the nuclear structure, leading to non-statistical inaccuracies in the average spin measurement for a particular nucleus? There are two empirical answers to this question, as follows. The first is addressed by the sensitivity analysis of the method to the inclusion or exclusion of non-yrast states (root-mean-square average difference 5.9\%). This implies a potential variation in the sensitivity of the method in the range of $0.3 \hbar-0.6 \hbar$ owing to the degree of incompleteness of the spectroscopic information.

A second estimation of the sensitivity, or accuracy, of the MSM can be obtained from analysis of the non-statistical variations of the data points around the fitted trends. The correlation coefficients obtained from the fits (see Extended Data Table 4) have values of typically $R^{2}=0.85$, implying that $\sim 15 \%$ of the variation is not accounted for by the fit. The statistical uncertainties account for an additional $~ 5 \%$ of the variation (for example, a root-mean-square average of $5.5 \%$ for the ${ }^{238} \mathrm{U}(n, f)$ reaction). Hence $10 \%$ of the variation of the variation remains unaccounted for. This can originate from three sources: second-order physics effects not included in the smooth parameterization, local spin mis-assignments/errors in the literature level scheme information, and local biases due to peculiarities of the local nuclear structure. The trend is measured over a range of approximately $4 \hbar-10 \hbar$ and hence we deduce that in the worst case, the sensitivity of the method is in the range $0.4 \hbar-1.0 \hbar$.

\section{Corrections applied in the MSM}

The MSM involves some further small corrections due to possible residual coincidences from $\beta$-decay, for the side feeding of the first $2^{+}$state, for the presence of isomeric states, and finally for statistical transitions from the continuum of unresolved non-yrast states. The correction methods are outlined below, followed by a description of how a transition intensity can be deduced indirectly, if it cannot be obtained directly or accurately fitted.

$\boldsymbol{\beta}$-decay. The experimental conditions were arranged to strongly suppress $\beta$-decay, achieved by tagging one fission fragment in the ionisation chamber for ${ }^{252} \mathrm{Cf}(\mathrm{SF})$ and by pulsation of the neutron beam with 400-ns period in the cases of ${ }^{232} \mathrm{Th}(n, f)$ and ${ }^{238} \mathrm{U}(n, f)$. Additional corrections were employed to remove any residual $\gamma$-ray coincidences from $\beta$-decay in the neutron-induced reaction data by subtracting an uncorrelated background. Background coincidence matrices were created using a pre-prompt trigger window 200 ns before a beam pulse of exactly the same size as the prompt window ( $80 \mathrm{~ns})$. Typically, the correction applied is very small, since these matrices contained only 1-2\% of the total counts of the prompt matrices, yet these subtractions are potentially important for the fragments closest to stability towards 
the end of the $\beta$-decay chains in fission (for example, ${ }^{98} \mathrm{Zr},{ }^{142} \mathrm{Ba}$, and so on). $\gamma$-ray coincidences from these nuclei will have larger components produced by this unwanted population process. If the correction is not applied this could lead to underestimates of the average spin in these particular nuclei owing to the presence of unwanted $\beta$-decay population pathways at lower spins.

Determination of the side-feeding of the first $2^{+}$state. The side feeding of the first excited state $\left(2^{+}\right.$in almost all even-even nuclei) cannot be measured directly from the $\gamma-\gamma$ coincidences of a particular nucleus. However, as noted in the original MSM paper ${ }^{29}$, it is possible to determine this side-feeding by selecting a strong transition in a partner fragment. The intensity ratio of the transition from the first excited state to the ground state, and the transition(s) feeding the first excited state can then be measured from the resulting spectrum and uncertainty determined. This ratio, labelled $G_{(2 / 4)}$ and shown in Extended Data Tables 1 and 2, is always greater than or equal to unity, since negative side-feedings are unphysical.

These ratios cannot be determined directly for all of the fragments studied, either because they and their partners are weakly populated, or because in some cases the transition energy is a doublet common to both fragment and partner, or to two neighbouring partners. We therefore fit the trends of $G_{(2 / 4)}$ ratios as a function of fragment mass in the light and heavy peaks for both the ${ }^{232} \mathrm{Th}$ and ${ }^{238} \mathrm{U}$, and use the fitted values with appropriate uncertainties. The $G_{(2 / 4)}$ ratios for the light peak show a gradual trend towards unity at the highest masses. However, the $G_{(2 / 4)}$ ratio in the heavy peak is initially high (around 2.5) near the doubly magic $\mathrm{Sn}$ shell closure and decreases rapidly towards unity with increasing mass. In the most extreme case, the side-feeding of the $2^{+}$state in ${ }^{132} \mathrm{Sn}$ populated in the ${ }^{238} \mathrm{U}(n, f)$ reaction accounts for some $60 \%$ of the total side-feeding intensity. This phenomenon may thus account for some of the observed anomaly at $Z=50$ when using the $\gamma$-ray coincidence method to determine fission yields ${ }^{47}$.

For the ${ }^{252} \mathrm{Cf}(\mathrm{SF})$ system it is not possible to deduce the $2^{+}$side-feedings from gating on the partner fragments since the partner fragment decays in-flight, so its transitions are Doppler broadened. In the case of ${ }^{252} \mathrm{Cf}(\mathrm{SF})$ we use $G_{(2 / 4)}$ ratio values deduced from the fits to the ${ }^{238} \mathrm{U}(n, f)$ data. The ${ }^{232} \mathrm{Th}(n, f) G_{(2 / 4)}$ ratios show similar variations with mass, but we assume that the ${ }^{238} \mathrm{U}(n, f)$ trends provide better estimates. This is preferable to assuming $2^{+}$state side-feeding values of zero for ${ }^{252} \mathrm{Cf}(\mathrm{SF})$, since it allows a better comparison of average spins in all three systems, but may necessarily introduce some small systematic bias.

Statistical transitions. The statistical side feeding transitions will also carry away a small quantity of angular momentum. In the original MSM paper calculations were used to estimate the average number of statistical transitions (2.5) and average angular momentum per transition $(0.4 \text { units })^{48}$. Here, we use these same values to facilitate comparison of results. These do not affect the shape of the observed saw-tooth distributions but will instead just shift them globally up or down in spin.

Isomeric states and delayed transitions. Calculating the average spin for a nucleus with a strongly populated isomer requires an additional step in the analysis. For isomeric transitions with lifetimes in the nanosecond to microsecond range, the $\gamma$-ray decay below the isomer can occur outside the trigger window and thus the $\gamma$-rays and their coincidences with states above the isomer are not observed, leading to an underestimate of the average spin if no correction is applied. The correction for ${ }^{252} \mathrm{Cf}(\mathrm{SF})$ data are very simple, since we can just increase the size of the prompt window from 100 ns to $4 \mu \mathrm{s}$. This results in an increase in the average spin of the most affected nuclei, ${ }^{132} \mathrm{Sn}$ and ${ }^{134} \mathrm{Te}$, of $12 \%$ and $14 \%$ respectively. No other nuclei show statistically significant (>2s.d.) increases in the deduced average spin for an extended coincidence window. Applying corrections for isomers in the ${ }^{232} \mathrm{Th}(n, f)$ and ${ }^{238} \mathrm{U}(n, f)$ datasets is more difficult. The prompt window is increased from 80 ns to $400 \mathrm{~ns}$, and the corresponding increase in spin for these key isomeric nuclei is measured. A further correction is then applied using an extrapolation to account for the missing isomeric coincidences beyond the 400-ns window.

For all three fissioning systems the nucleus ${ }^{130} \mathrm{Sn}$ presents a unique

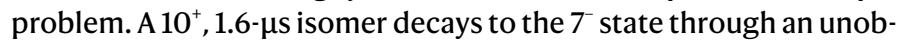
servable $96-\mathrm{keV}$ transition and this $7^{-}$state has a half-life of $1.7 \mathrm{~min}$. Hence, there is missing intensity for this nucleus. We include ${ }^{130} \mathrm{Sn}$ in our data, but acknowledge the existence of a potential systematic error in the calculation of the average spin for this particular case. However, since the neighbouring ${ }^{132} \mathrm{Sn}$ also has a similar high-spin isomer, and the inclusion or exclusion of these decays changes the average spin by only $12 \%$, we assume that the systematic underestimate of average spin for ${ }^{130} \mathrm{~S} n$ will be smaller than the statistical uncertainties.

Redundancy and the indirect deduction of intensity information. As mentioned previously, there is some redundancy in the $\gamma$-ray transition intensity, given that we measure a cumulative intensity flow. This redundancy can be exploited to recover missing intensity information in the rare case that it is necessary. A problem that can occur during a two-dimensional coincidence analysis is that occasionally, certain coincidences or transitions can be obscured by the presence of others if they are too close in energy. This generally presents more of a difficulty for a small number of weak transitions in nuclei with the lowest production yields. For example, there is a strong background of random 511-keV $\gamma$-rays from electron-positron annihilation. For a nucleus that has a weak transition very close to this energy it is often impossible to measure its intensity directly owing to the large statistical fluctuations present after subtraction of this dominating background.

If such doublets or multiplets are too close in energy to resolve by two-dimensional peak fitting, information on the intensity of the obscured transition can still be recovered from the intensity flows into and out of its initial and final states. For the general case, the intensity, $G$, of a $\gamma$-ray transition between initial state $A$ and final state $B$ can never be smaller than the intensity balance into state $A$ and never be larger than the intensity balance out of state $B$, since this would result in negative side-feedings for $A$ or $B$, which is unphysical.

If $G_{\mathrm{Ai}}$ and $G_{\mathrm{Ao}}$ are the measured ingoing and outgoing intensities from state $A$, and $G_{\mathrm{Bi}}$ and $G_{\mathrm{Bo}}$ those for state $B$, then the intensity of the missing transition intensity, $G_{x}$, must obey the following relation $\left(G_{\mathrm{Ai}}-G_{\mathrm{Ao}}\right) \geq G_{\mathrm{x}} \geq\left(G_{\mathrm{Bo}}-G_{\mathrm{Bi}}\right)$. The best estimate of the intensity $G_{\mathrm{x}}$ is thus the average $\left(\left(G_{\mathrm{Ai}}-G_{\mathrm{Ao}}\right)+\left(G_{\mathrm{Bo}}-G_{\mathrm{Bi}}\right)\right) / 2$ of the upper and lower bound and implies that the side-feedings of state $A$ and state $B$ are equal. The impact of deducing a $\gamma$-ray intensity on the average spin measured for a particular nucleus is negligible because the measurement integrates the intensity information from many transitions and the deduced intensity is usually a very good estimate of the real intensity.

\section{Potential sources of bias}

The MSM has several sources of potential bias outlined below.

Level schemes. Since we are observing neutron-rich nuclei far from stability in these experiments, the level schemes in the literature (see the ENSDF databases ${ }^{46}$ ) may have spin assignments of certain levels which are only tentative, and in some cases incorrect by 1 or 2 spin units. This may have a small impact on the average spin extracted for a particular nucleus. However, the main side-feeding branches usually occur at lower spins with the $2^{+}, 4^{+}$and $6^{+}$yrast levels accounting for a large fraction of the total side-feeding intensity in most cases. These states are usually well measured with unequivocal spin assignments. Mis-assignment of the spins of certain states may either slightly lower or slightly raise the average spin deduced for a particular nucleus. However, the effect will be local, can occur in either direction, and will be confined to a particular nucleus. No global systematic effect is expected. 
Ground state feeding. The direct side-feeding of the ground state is impossible to measure using $\gamma$-ray spectroscopy. However, we can estimate it from an extrapolation of the spin distribution toward zero. Extended Data Fig. 4 shows how this extrapolation is performed from fits to the spin distribution associated with each data point. The extracted $0^{+}$feeding is given an appropriately large relative uncertainty. The $0^{+}$feeding is typically $3-5 \%$ of the total side-feeding intensity in most cases, but increases in the vicinity of closed-shell nuclei (up to $18 \%$ in the case of $\left.{ }^{132} \mathrm{Sn}\right)$. The impact of this correction on the average spin values results in a slight lowering, which is smaller than the statistical errors. However, for ${ }^{132} \mathrm{Sn}$ and its near neighbours with substantial $0^{+}$ feeding the average spin values drop considerably more by typically $0.3 \hbar$. Performing this correction has no impact on the conclusions.

Non-inclusion of weaker transitions. A potential bias in extraction of average spin could occur as a function of the fragment yield. Nuclei that are more weakly populated in general may have fewer observable transitions and levels available for inclusion in the weighted sum. However, we conclude that the MSM method is very insensitive to the inclusion or exclusion of non-yrast levels. Provided transitions from levels in the yrast sequence are visible, a reliable extraction of average spin can be made. The non-observation of weak transitions at the top of the yrast sequence has little impact on the final result, since if the transitions are weak at this point, the side-feedings are also weak and contribute little to the result. To quantify this potential bias, average spins in the 15 most strongly populated nuclei were recalculated after fitting only the yrast sequences and ignoring the presence of all other non-yrast states and transitions. The root-mean-square difference between the two sets of values was found to be $5.9 \%$. The transition rates of statistical side-feeding transitions are orders of magnitude faster than the intra-yrast cascade transitions and this probably accounts for why the difference is small. Finally, if the measured average spins for all nuclei studied are plotted against fragment yields, the two quantities are seen to be almost entirely uncorrelated.

The trigger condition. For the case of the ${ }^{252} \mathrm{Cf}(\mathrm{SF})$, the trigger condition was an anode signal of the ionization chamber corresponding to detection of one fragments in flight, with the other stopped rapidly in the backing of the sample. This gives a clean, unambiguous signal that a fission has occurred. For the ${ }^{232} \mathrm{Th}(n, f)$ and ${ }^{238} \mathrm{U}(n, f)$ reactions the fission discrimination is less perfect. Although the beam pulsation allows discarding of events which are uncorrelated in time, a minimum multiplicity condition is also used in the prompt trigger window. This is essential to distinguish fission from the complex background of other low-multiplicity processes that also occur during the beam pulse, such as inelastic scattering ${ }^{238} \mathrm{U}\left(n, n^{\prime}\right),{ }^{27} \mathrm{Al}\left(n, n^{\prime}\right),{ }^{72,73,74,76} \mathrm{Ge}\left(n, n^{\prime}\right)$, $p\left({ }^{7} \mathrm{Li}^{7}{ }^{7} \mathrm{Li}^{\prime}\right)$ Coulomb excitation of the primary beam, and the intrinsic activity of the targets. The intrinsic activity is a particular problem for the ${ }^{232} \mathrm{Th}$ target, as a fraction of its decay also occurs during the prompt beam pulse. Since the majority of $\gamma$-rays detected during the experiment come from these low-multiplicity processes, a minimum trigger condition of $M_{\gamma} \geq 3$ is essential in order to preferentially select fission events. For the best discrimination the trigger condition should be placed at even higher multiplicities but we have deliberately kept it at 3 to minimize any potential trigger biases, even though this results in larger backgrounds. From the ${ }^{252} \mathrm{Cf}(\mathrm{SF})$ data it is possible to study the impact of the multiplicity trigger condition on the results. Raising the minimum trigger condition from the ionization chamber fission tag from a minimum multiplicity of two to three has no noticeable impact on the measured average spins. Effects are, however, observed at higher-multiplicity conditions. A global increase in the average spin for all nuclei of around one spin unit is observed for an increase of around 4 units in detected multiplicity. This correlation is completely expected and gives us further confidence in the key observation of this paper, namely the absence of spin correlations between fragment partners. The reason the slope of the correlation of average spin with detected multiplicity is not larger is mostly due to the imperfections in the $v$-Ball calorimeter (68\% efficiency for detecting a single $\gamma$-ray at $1 \mathrm{MeV}$ ). An event of detected multiplicity (or fold) of 4 will thus have sizeable contributions from emitted multiplicities of $4,5,6,7$ and 8 .

Derivation of the spin parameterization from statistical theory. The expected probability distribution, $P(I)$, of angular momenta, $I$, for an excited nucleus, following the work of Hans Bethe ${ }^{35}$, is:

$$
P\left(I \mid \sigma^{2}\right)=\frac{2 I+1}{2 \sigma^{2}} \exp \left(-\frac{(I+1 / 2)^{2}}{2 \sigma^{2}}\right)
$$

where $\sigma$ is known as a spin-cutoff parameter describing the breadth of this distribution. In the Fermi gas model, the spin cut-off parameter depends directly on the nuclear temperature, $T$, and is related to the excitation energy $E_{\mathrm{x}}$ and the level density parameter, $a$.

$$
T=\sqrt{\frac{E_{\mathrm{x}}}{a}}
$$

In this model, the spin cut-off parameter is then usually defined as the product of the rigid body moment of inertia, $\mathcal{I}_{\text {rigid }}$ and the temperature:

$$
\sigma^{2}=\mathcal{I}_{\text {rigid }} T
$$

where for a spheroidal nucleus

$$
\mathcal{I}_{\text {rigid }}=\frac{2}{5} A_{\mathrm{F}} R^{2}
$$

so $\mathcal{I}_{\text {rigid }} \propto A_{\mathrm{F}}^{5 / 3}$. Using a level density parameter $a$ that is proportional to $A_{\mathrm{F}}$, the variation in the spin cut-off parameter with fragment mass can then be defined in the following way:

$$
\sigma^{2} \propto \sqrt{E_{\mathrm{x}}} A_{\mathrm{F}}^{7 / 6}
$$

If we assume that the excitation energy of the fragment is proportional to the mass of the nucleons from the ruptured neck (that is, $E_{\mathrm{x}} \propto A_{\mathrm{N}}$ ), we obtain this final parameterization based on statistical theory, which can be used to fit our average spin data in Fig. 1:

$$
I=c A_{\mathrm{N}}^{1 / 4} A_{\mathrm{F}}^{7 / 12}
$$

where $I \approx 1.15 \sigma$. This smooth parameterization of the mass dependence with only one free parameter can be used as a fitting function, analogous to the smooth fitting of nuclear mass variations with the Weissacker formula ${ }^{49}$. An extended theoretical description would also have additional local variations in $E_{x}\left(A_{\mathrm{F}}\right)$, level density $a\left(A_{\mathrm{F}}\right)$ and $\mathcal{I}_{\text {rigid }}\left(A_{\mathrm{F}}\right)$ owing to structure effects. However, the smooth functional dependence of $I\left(A_{\mathrm{F}}\right)$ captures the major ingredients of the variation.

Monte Carlo code for correlated fragment spins. For the data presented in Fig. 2, a dispersion propagation Monte Carlo code was developed to understand what experimental slopes we would expect to see if the intrinsic angular momentum were generated by pre-scission mechanisms that produce correlated spins at scission (that is, the precise width and location of case 'Pre-scission' in the inset of Fig. 2). The emission of neutrons and statistical $\gamma$-rays in each fragment will have a de-correlating effect on any spin measurements carried out at or near the yrast lines. The precise and only purpose of this code is to propagate realistic spin dispersions from scission to yrast simultaneously in both fragments owing to emission of neutrons and statistical $\gamma$ rays. It allows for (i) complete user control over the spin distribution parameters at 
scission, (ii) total control over the widths of the spin dispersions due to emission of both neutrons and $\gamma$-rays, and (iii) the ability to output the resulting spin distribution observed at yrast when setting conditions on the spin distribution in the partner fragment.

Dispersions in spin due to neutron emission were taken as random $\pm 0.5 \hbar$ per emitted neutron. To obtain statistical $\gamma$-ray spin dispersions, the RAINIER $\operatorname{code}^{50}$ was used to fully model the $\gamma$-decay of several representative spherical and deformed fission fragments. Typical statistical $\gamma$-ray spin dispersion distributions with a width of around $\pm 1.5 \hbar$ were then imported into our Monte Carlo code.

The placement of different gating conditions at yrast on one fragment could then be simulated and the effect on the resulting spin distribution at yrast in the partner fragment could be determined (see Extended Data Fig. 3). A simulated experimental relationship between the gating condition at yrast in one fragment and the 'measured' average spin in the other could then be probed and an 'experimental' slope deduced. With these tools, a sensitivity analysis of the results to the parameters of the initial spin distribution and neutron $/ \gamma$-dispersions could be performed. The blue band in the inset of Fig. 2 for case 'Pre-scission') gives a range of expected slopes (0.4-0.6) for fully correlated spins at scission for reasonable variations of these parameters. The conclusion is that statistical emission will slightly weaken any spin correlations present at scission but will not destroy them.

We also note that similar experimental data exists for 1n partners. These are not shown in Fig. 2, which would have become much too cluttered. However, the results obtained are similar, with comparable slopes observed.

An extension of this Monte-Carlo code to include the $\gamma$-ray spectrometer granularity and detection efficiency was also developed. Here, the concern was that demanding observation of a 'high spin' state in one fragment might reduce the overall efficiency of detection owing to potential biases towards higher-multiplicity events involving many detectors. Since v-Ball is a highly granular array (106 separate Ge elements at large distances) this effect on the expected slopes proved to be completely negligible. However, for the case of very close-packed detector arrays (for example, 6 clover detectors placed in cubic geometry) small negative slopes could arise from such biases, suggesting an artificial anti-correlation between fragment spins.

\section{Data availability}

All data from which the conclusions of this paper are drawn are contained within this manuscript. All other data can be made available on reasonable request. The large quantities of raw data (approximately $120 \mathrm{~Tb}$ ) are shared within the $\mathrm{v}$-Ball Collaboration on servers at the CNRS-IN2P3 Centre de Calcul in Lyon (https://cc.in2p3. fr). The ALTO facility of the IJC Laboratory has a transparent data management policy that complies with the relevant European directives on open data (https://ec.europa.eu/digital-single-market/en/ european-legislation-reuse-public-sector-information). Raw data from the $v$-Ball Collaboration will be made publicly available after a period of 5 years. Source data are provided with this paper.

\section{Code availability}

All codes used in the data analysis can be made available on reasonable request.

41. Etasse, D. et al. Fast Acquisition System for nuclEar Research (FASTER); http://faster.in2p3. fr (2013).

42. Hamilton, J. et al. New insights from studies of spontaneous fission with large detector arrays. Prog. Part. Nucl. Phys. 35, 635 (1995).
43. Smith, A. G. et al. Spectroscopy of neutron-rich nuclei populated in the spontaneous fission of ${ }^{252} \mathrm{Cf}$ and ${ }^{248} \mathrm{Cm}$. AIP Conf. Proc. 481, 283 (1999).

44. Agostinelli, S. et al. GEANT IV-a simulation toolkit. Nucl. Instrum. Meth. Phys. Res. A 506, 250-303 (2003).

45. Radford, D. C. ESCL8R and LEVIT8R: software for interactive graphical analysis of HPGe coincidence data sets. Nucl. Instrum. Meth. Phys. Res. A 361, 297-305 (1995).

46. Tuli, J. K. Evaluated nuclear structure data file. Nucl. Instrum. Meth. Phys. Res. A 369 506-510 (1996).

47. Wilson, J. N. et al. Anomalies in the charge yields of fission fragments from the ${ }^{238} \cup(n, f)$ reaction. Phys. Rev. Lett. 118, 222501 (2017).

48. Pühlhofer, F. On the interpretation of evaporation residue mass distributions in heavy-ion induced fusion reactions. Nucl. Phys. A 280, 267-284 (1977).

49. Weizsäcker, C. F. Zur Theorie der Kernmassen. Z. Phys. 96, 431-458 (1935).

50. Kirsch, L. E. \& Bernstein, L. A. RAINIER: a simulation tool for distributions of excited nuclear states and cascade fluctuations. Nucl. Instrum. Meth. Phys. Res. A 892, 30-40 (2018).

51. England, T. R. \& Rider, B. F. Evaluation And Compilation Of Fission Product Yields. Los Alamos Report LA-UR-94-3106, ENDF-349, https://www-nds.iaea.org/endf349/ (International Atomic Energy Agency (IAEA) Nuclear Data Services, 1993).

Acknowledgements We thank the staff of the ALTO facility of the IJC Laboratory, Orsay, for providing the intense, precisely focused ${ }^{7}$ Li primary beams for very long periods, thus permitting the collection of large datasets with the v-Ball spectrometer. We thank W. Nazerewicz, S. Åberg and O. Serot for discussions of fission theory; we also thank S. Åberg and O. Serot for assistance with the theoretical interpretation of our experimental results. We thank G. Kessedijan for assistance with variance-covariance calculations. Finally, we thank the Gammapool international consortium for the loan of the germanium clover detectors used to construct the spectrometer. This work was supported by the IN2P3/CNRS, France, the STFC UK Nuclear Data Network, the STFC (grants ST/L005743/1 and ST/PO05314) (PHR), the Marion Redfearn Trust (RCL). P.H.R., M.B., A. Boso and P.I. acknowledge support from the UK Department of Business, Energy and Industrial Strategy (BEIS) via the National Measurement System. P.K., P.-A.S. and J.W. acknowledge the support from BMBF under grant NuSTAR.DA 05P15RDFN1. Funding from the HORIZON2O2O programme of the European Commission is acknowledged for Transnational Access to the ALTO facility under the Integrated Infrastructure Initiative, European Nuclear Science and Applications Research 2 (ENSAR2), grant agreement number 654002 . A. Blazhev, R.-B.G. and N.W. acknowledge support by the German Research Foundation (DFG grant BL 1513/1-1). L.F., V.V., J.B. and V.S.-T. acknowledge funding from the Spanish MINECO via FPA2015-65035-P and RTI2018-098868-B-IOO. A.A. acknowledges funding Spanish MINECO via FPA2017-83946-C2-1-P and Ministerio de Ciencia e Innovacion grant PID2019-104714GB-C21. B.F. acknowledges funding from the Polish National Science Centre under contracts 2014/14/M/ST2/00738 and 2013/08/M/ST2/00257. M.P. acknowledges funding from the Polish National Science Centre under contracts UMO-2019/33/N/ST2/03023, UMO-202O/36/T/ST2/00547 and A.K., K.M. and E.A. under contract UMO-2015/18/E/ ST2/00217. D.G. acknowledges funding from the Norges Forskningsråd (Research Council of Norway) 263030 . S.L., G.B., C.P., S.Z., L.W.I, A.G. and S.B. acknowledge funding from the Italian Istituto Nazionale di Fisica Nucleare (INFN)

Author contributions J.N.W. participated in the construction of the v-Ball spectrometer, contributed to the experimental data taking, organised the v-Ball international collaboration, performed the analysis work presented here and wrote the main body of the paper. D.G. helped with experimental and theoretical discussions, interpretation of results, manuscript preparation and resubmissions, calculations, plots and bibliography. D.T. constructed the spectrometer, calibrated and optimized the spectrometer, kept the spectrometer running, contributed to the experimental data taking, performed data processing of the large quantities of triggerless data and helped distribute it to the collaboration. M.L. organized the v-Ball project, led the construction of the spectrometer, organised the experimental campaign, kept the spectrometer running, contributed to the experimental data taking and measured v-Ball performances. M.R., N.J., R.C., G.H., R.L. and R.-B.G. helped with the cabling of the v-Ball spectrometer, supported the running of the spectrometer (filling with liquid nitrogen, monitoring detectors, and so on), calibrated and optimized the spectrometer, contributed to the experimental data taking and performed offline data analysis. D.E. developed and helped deploy the digital electronics used for the v-Ball Data Acquisition System). L.G. developed and deployed the ${ }^{252} \mathrm{Cf}$ ionization chamber. S.O., C.S., T.K., P.H.R., A. Blazhev, N.W., S.L., B.F., A.A., M.F., L.F. and others contributed to the theoretical discussions and interpretation of results. S.S. helped with organization, discussions and interpretation, bibliography and manuscript preparation. F.Z. carried out fragment decay simulations using the RAINIER code. All listed collaborators helped keep the experiment, the spectrometer and the data acquisition systems running over the period of 7 weeks during which the data were collected.

Competing interests The authors declare no competing interests.

\section{Additional information}

Supplementary information The online version contains supplementary material available at https://doi.org/10.1038/s41586-021-03304-w.

Correspondence and requests for materials should be addressed to J.N.W.

Peer review information Nature thanks Cedric Simenel, Patrick Talou and the other, anonymous, reviewer(s) for their contribution to the peer review of this work. Peer reviewer reports are available.

Reprints and permissions information is available at http://www.nature.com/reprints. 


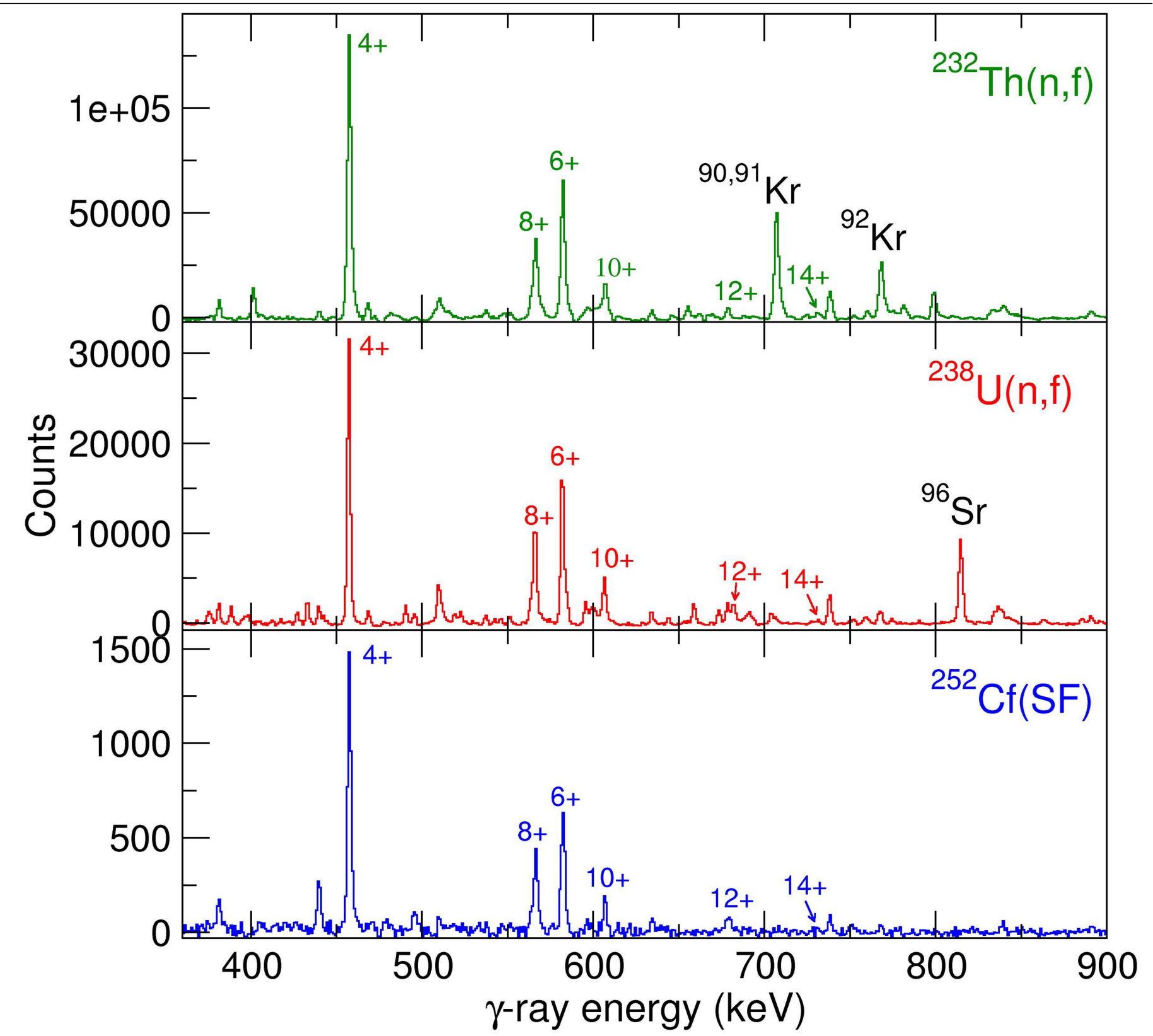

Extended Data Fig. $1 \mid \boldsymbol{\gamma}$-ray coincidence spectra for ${ }^{140} \mathrm{Xe}$. Spectra are gated by the $2^{+} \rightarrow 0^{+}$transition for the three different fissioning systems studied in this work. The spins of states emitting the yrast sequence of transitions are marked. Strong $\gamma$-rays from the binary partner fragments are indicated. $\gamma$-rays from

not visible owing to Doppler broadening. The ${ }^{252} \mathrm{Cf}(\mathrm{SF})$ spectrum has many fewer counts, but similar experimental sensitivity is achieved owing to the elimination of backgrounds from other processes by direct detection of the fission fragment in the ionization chamber with the $\gamma-\gamma$ coincidences. 


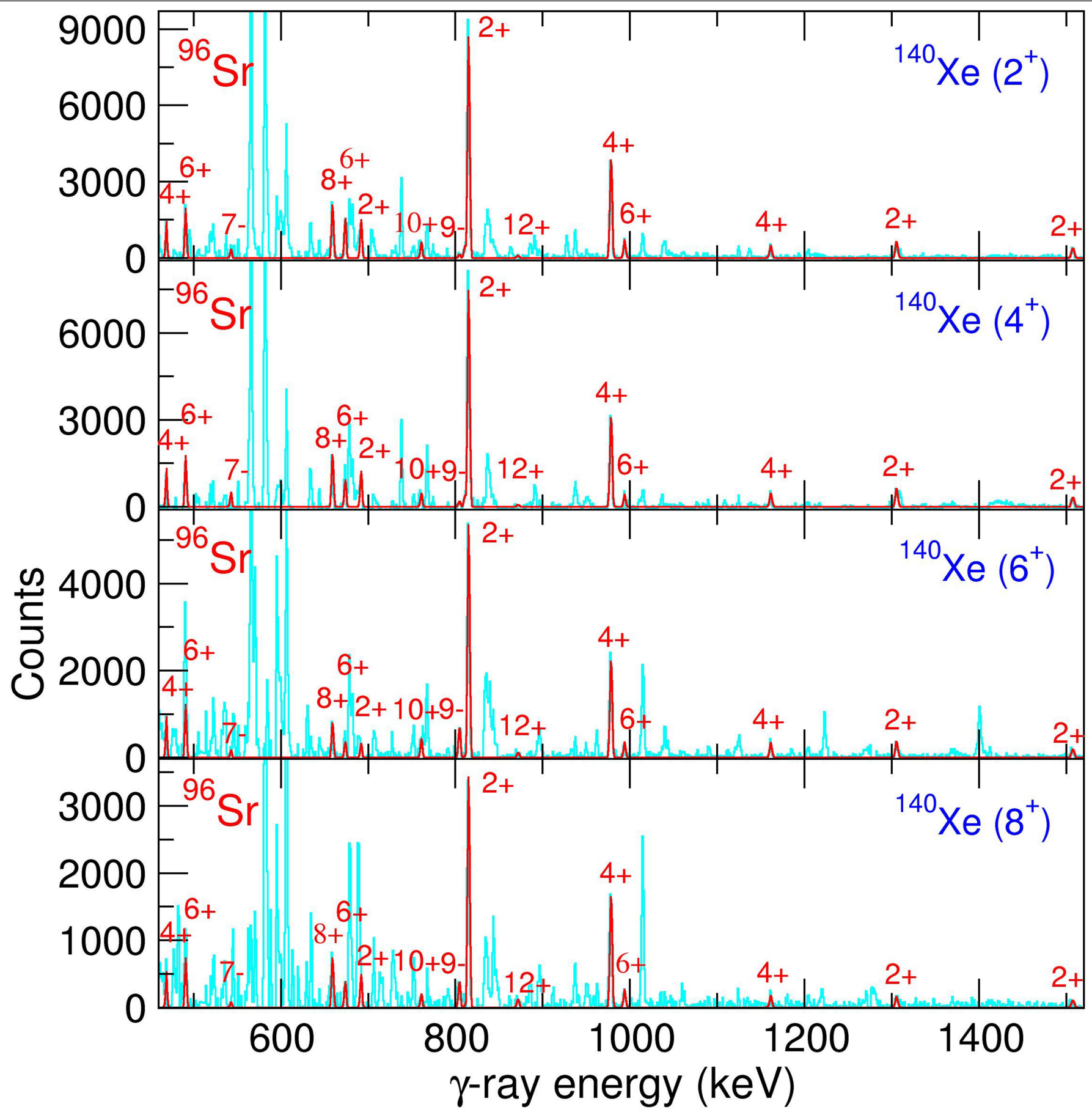

Extended Data Fig. 2 | Coincident $\gamma$-ray spectra from the ${ }^{238} \mathrm{U}(n, f)$ reaction gated on transitions from ${ }^{100} \mathrm{Xe}$ emitted from states of increasing spin. The fits to transitions decaying out of specific states of the partner nucleus ${ }^{96} \mathrm{Sr}$ are shown in red. The $492-\mathrm{keV}$ transition from the $6+$ state in ${ }^{96} \mathrm{Sr}$ in the third panel is deduced from its neighbours rather than fitted, owing to contamination. The intensity pattern is not observed to vary and the average spins in ${ }^{96} \mathrm{Sr}$ show no notable changes. The relationships between partner spins for several more nuclei are shown in Fig. 2. 


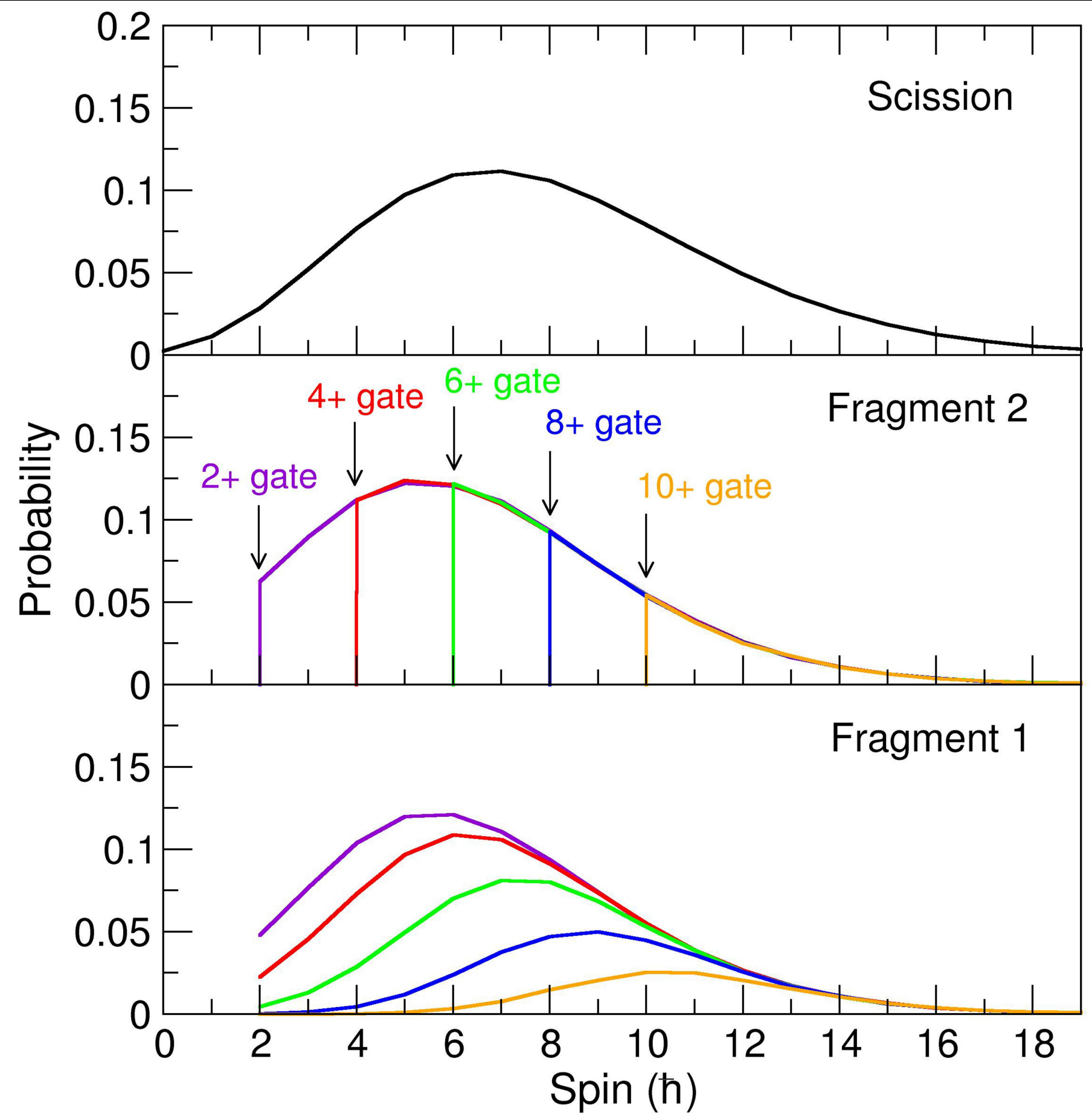

Extended Data Fig. 3 | Monte Carlo simulations of events with correlated spins at scission. Placing conditions on the minimum spin at yrast of events in fragment 1 affects the yrast distributions of event spins in fragment 2 . 


\section{Article}

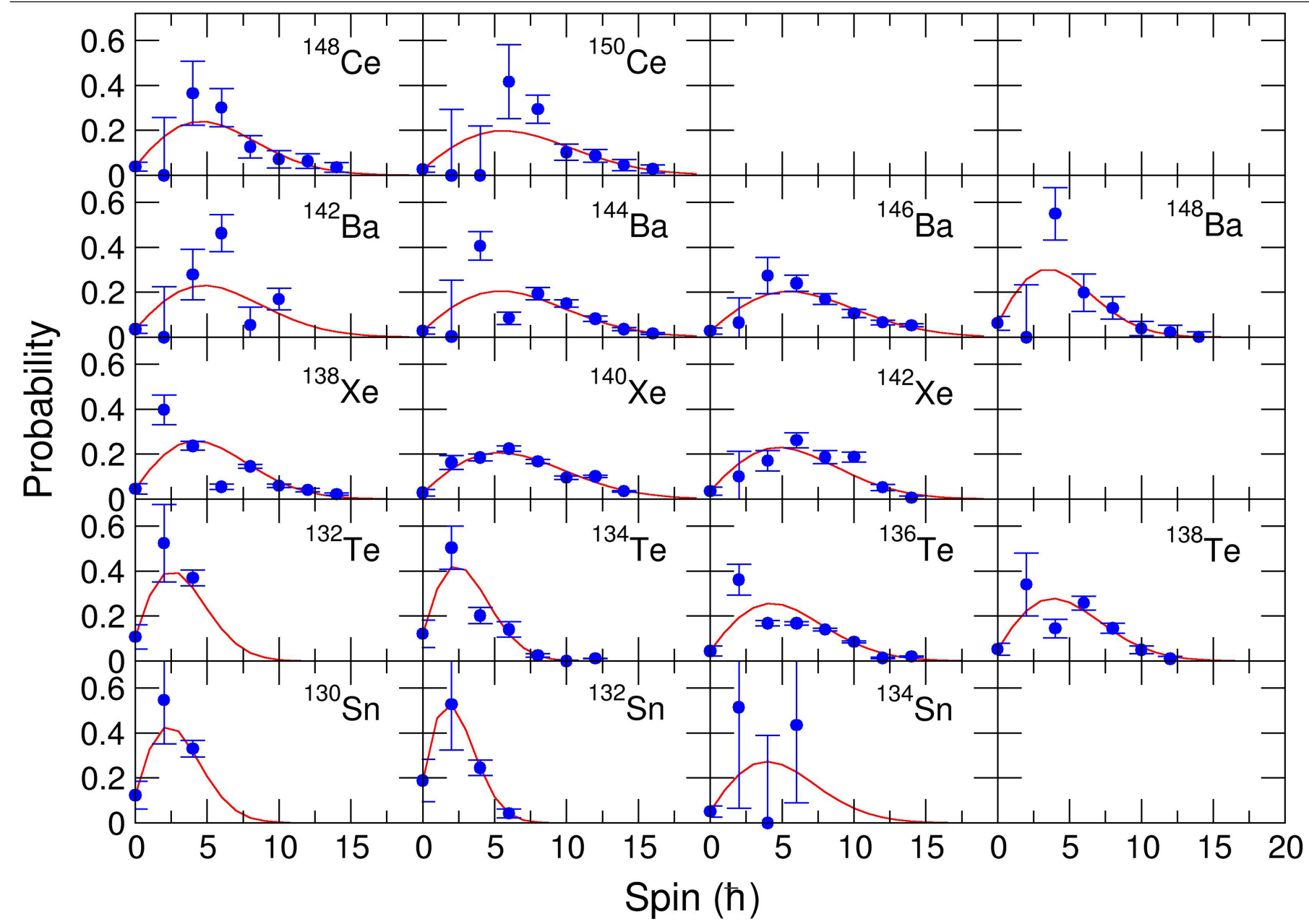

Extended Data Fig. 4 | Examples of experimental spin distributions for a range of nuclei observed in the ${ }^{238} U(n, f)$ reaction. Statistical uncertainties are shown. To eliminate the odd-even staggering effect and facilitate easy visualization, side-feedings of odd spins are redistributed equally between the two neighbouring even spins. The red curves are fits to the experimental data with one free parameter and are used to extract 0 + side-feedings via an iterative procedure. 
Extended Data Table $\left.1\right|^{232} \mathrm{Th}(n, f)$ average spin data

\begin{tabular}{lllllll}
\hline Nucleus & $\langle\mathrm{>}\rangle$ & $\sigma_{<\mid>}$ & $\mathrm{G}_{(2 / 4)}$ & $\sigma_{\mathrm{G}(2 / 4)}$ & $0^{+}$feed & Yield(\%) \\
\hline${ }^{82} \mathrm{Ge}$ & 4.10 & 0.10 & - & - & 0.11 & 0.64 \\
${ }^{84} \mathrm{Ge}$ & 3.92 & 0.78 & - & - & 0.09 & 0.32 \\
${ }^{84} \mathrm{Se}$ & 3.85 & 0.03 & - & - & 0.17 & 1.09 \\
${ }^{86} \mathrm{Se}$ & 4.26 & 0.05 & 1.13 & 0.16 & 0.11 & 4.68 \\
${ }^{8} \mathrm{Se}$ & 4.41 & 0.16 & - & - & 0.10 & 2.21 \\
${ }^{88} \mathrm{Kr}$ & 4.75 & 0.14 & 1.99 & 0.35 & 0.12 & 0.85 \\
${ }^{90} \mathrm{Kr}$ & 4.72 & 0.04 & - & - & 0.08 & 5.34 \\
${ }^{9} \mathrm{Kr}$ & 5.10 & 0.12 & 1.94 & 0.19 & 0.07 & 3.92 \\
${ }^{94} \mathrm{Kr}$ & 6.02 & 0.52 & - & - & 0.05 & 0.60 \\
${ }^{92} \mathrm{Sr}$ & 4.05 & 0.61 & 1.57 & 0.41 & 0.09 & 0.20 \\
${ }^{94} \mathrm{Sr}$ & 4.44 & 0.11 & 1.44 & 0.04 & 0.10 & 2.04 \\
${ }^{96} \mathrm{Sr}$ & 5.52 & 0.09 & 1.07 & 0.10 & 0.06 & 3.54 \\
${ }^{98} \mathrm{Sr}$ & 6.01 & 0.20 & - & - & 0.06 & 1.32 \\
${ }^{98} \mathrm{Zr}$ & 6.40 & 0.65 & - & - & 0.04 & 0.37 \\
${ }^{100} \mathrm{Zr}$ & 6.07 & 0.91 & - & - & 0.03 & 0.88 \\
${ }^{130} \mathrm{Sn}$ & 3.29 & 0.68 & - & - & 0.15 & 0.84 \\
${ }^{132} \mathrm{Sn}$ & 3.64 & 0.15 & 2.64 & 1.40 & 0.19 & 1.54 \\
${ }^{132} \mathrm{Te}$ & 3.38 & 0.48 & - & - & 0.13 & 0.35 \\
${ }^{134} \mathrm{Te}$ & 3.44 & 0.20 & 3.51 & 0.48 & 0.18 & 3.11 \\
${ }^{136} \mathrm{Te}$ & 4.83 & 0.13 & 2.18 & 0.15 & 0.07 & 3.44 \\
${ }^{138} \mathrm{Te}$ & 4.74 & 0.32 & - & - & 0.07 & 0.76 \\
${ }^{138} \mathrm{Xe}$ & 4.53 & 0.38 & - & - & 0.13 & 2.08 \\
${ }^{140} \mathrm{Xe}$ & 7.70 & 0.62 & 1.25 & 0.10 & 0.02 & 5.73 \\
${ }^{142} \mathrm{Xe}$ & 7.60 & 0.24 & 1.12 & 0.15 & 0.04 & 2.25 \\
${ }^{142} \mathrm{Ba}$ & 5.87 & 0.73 & 1.27 & 0.14 & 0.05 & 0.64 \\
${ }^{144} \mathrm{Ba}$ & 7.49 & 0.27 & 1.00 & 0.04 & 0.03 & 4.49 \\
${ }^{146} \mathrm{Ba}$ & 7.03 & 0.31 & 1.12 & 0.11 & 0.04 & 2.76 \\
${ }^{148} \mathrm{Ce}$ & 7.70 & 0.70 & 1.00 & 0.25 & 0.03 & 0.56 \\
${ }^{150} \mathrm{Ce}$ & 8.74 & 0.65 & - & - & 0.03 & 0.41 \\
\hline & & & & & & \\
\hline
\end{tabular}

Measured values are given for average spin, $\left\langle l>\right.$, its associated uncertainty $\sigma_{\langle<\rangle}$, the measured intensity ratio $G_{(2 / 4)}$ and its associated uncertainty, $\sigma_{G(2 / 4)}$, the $\mathrm{O}^{+}$feeding value and the fragment yield. Fragment yields are taken from the evaluated nuclear data files ENDF.BVII ${ }^{51}$. 


\section{Article}

Extended Data Table $\left.2\right|^{238} U(n, f)$ average spin data

\begin{tabular}{|c|c|c|c|c|c|c|}
\hline Nucleus & $<1>$ & $\sigma_{<1>}$ & $\mathrm{G}_{(2 / 4)}$ & $\sigma_{\mathrm{G}(2 / 4)}$ & $0^{+}$feed & Yield(\%) \\
\hline${ }^{82} \mathrm{Ge}$ & 4.41 & 0.67 & - & - & 0.09 & 0.12 \\
\hline${ }^{84} \mathrm{Se}$ & 3.98 & 0.18 & - & - & 0.16 & 0.17 \\
\hline${ }^{86} \mathrm{Se}$ & 4.88 & 0.24 & 1.48 & 0.30 & 0.07 & 0.84 \\
\hline${ }^{88} \mathrm{Se}$ & 4.72 & 0.36 & 1.20 & 0.23 & 0.10 & 0.54 \\
\hline${ }^{88} \mathrm{Kr}$ & 4.37 & 0.32 & - & - & 0.10 & 0.37 \\
\hline${ }^{90} \mathrm{Kr}$ & 4.76 & 0.10 & - & - & 0.08 & 1.85 \\
\hline${ }^{92} \mathrm{Kr}$ & 5.21 & 0.08 & 1.66 & 0.37 & 0.06 & 2.50 \\
\hline${ }^{94} \mathrm{Kr}$ & 5.27 & 0.42 & 1.60 & 0.31 & 0.05 & 0.73 \\
\hline${ }^{94} \mathrm{Sr}$ & 4.76 & 0.10 & 1.22 & 0.09 & 0.09 & 1.51 \\
\hline${ }^{96} \mathrm{Sr}$ & 5.11 & 0.05 & 1.31 & 0.06 & 0.06 & 4.13 \\
\hline${ }^{98} \mathrm{Sr}$ & 6.08 & 0.12 & 0.95 & 0.14 & 0.04 & 2.27 \\
\hline${ }^{98} \mathrm{Zr}$ & 5.98 & 0.32 & 0.94 & 0.10 & 0.06 & 0.49 \\
\hline${ }^{100} \mathrm{Zr}$ & 6.14 & 0.11 & - & - & 0.05 & 3.30 \\
\hline${ }^{102} \mathrm{Zr}$ & 6.12 & 0.08 & 1.11 & 0.12 & 0.05 & 4.09 \\
\hline${ }^{104} \mathrm{Zr}$ & 5.71 & 0.24 & - & - & 0.06 & 1.01 \\
\hline${ }^{102} \mathrm{Mo}$ & 7.87 & 0.50 & - & - & 0.02 & 0.08 \\
\hline${ }^{104} \mathrm{Mo}$ & 6.53 & 0.17 & 1.45 & 0.30 & 0.04 & 1.08 \\
\hline${ }^{130} \mathrm{Sn}$ & 3.46 & 0.40 & - & - & 0.12 & 1.65 \\
\hline${ }^{132} \mathrm{Sn}$ & 3.75 & 0.25 & 2.84 & 0.64 & 0.19 & 1.88 \\
\hline${ }^{134} \mathrm{Sn}$ & 4.65 & 1.16 & - & - & 0.05 & 0.18 \\
\hline${ }^{132} \mathrm{Te}$ & 3.56 & 0.40 & - & - & 0.10 & 0.47 \\
\hline${ }^{134} \mathrm{Te}$ & 4.26 & 0.17 & 2.33 & 0.24 & 0.12 & 3.95 \\
\hline${ }^{136} \mathrm{Te}$ & 5.82 & 0.06 & 1.61 & 0.11 & 0.04 & 3.53 \\
\hline${ }^{138} \mathrm{Te}$ & 5.60 & 0.20 & 1.56 & 0.21 & 0.05 & 0.55 \\
\hline${ }^{138} \mathrm{Xe}$ & 5.62 & 0.18 & - & - & 0.05 & 2.04 \\
\hline${ }^{140} \mathrm{Xe}$ & 7.41 & 0.10 & 1.16 & 0.03 & 0.03 & 4.04 \\
\hline${ }^{142} \mathrm{Xe}$ & 7.53 & 0.21 & 1.12 & 0.11 & 0.04 & 1.53 \\
\hline${ }^{142} \mathrm{Ba}$ & 7.02 & 0.76 & - & - & 0.04 & 0.69 \\
\hline${ }^{144} \mathrm{Ba}$ & 7.94 & 0.16 & 1.43 & 0.35 & 0.03 & 2.46 \\
\hline${ }^{146} \mathrm{Ba}$ & 7.60 & 0.22 & 1.07 & 0.06 & 0.03 & 1.98 \\
\hline${ }^{148} \mathrm{Ba}$ & 6.10 & 0.42 & - & - & 0.06 & 0.25 \\
\hline${ }^{148} \mathrm{Ce}$ & 7.24 & 0.59 & - & - & 0.04 & 0.75 \\
\hline${ }^{150} \mathrm{Ce}$ & 9.01 & 0.54 & - & - & 0.03 & 0.86 \\
\hline
\end{tabular}

Measured values are given for average spin, $\langle l\rangle$, its associated uncertainty $\sigma_{<\mid>}$, the measured intensity ratio $G_{(2 / 4)}$ and its associated uncertainty, $\sigma_{G(2 / 4)}$, the $O^{+}$feeding value and the fragment yield. Fragment yields are taken from the evaluated nuclear data files ENDF.BVII ${ }^{51}$. 


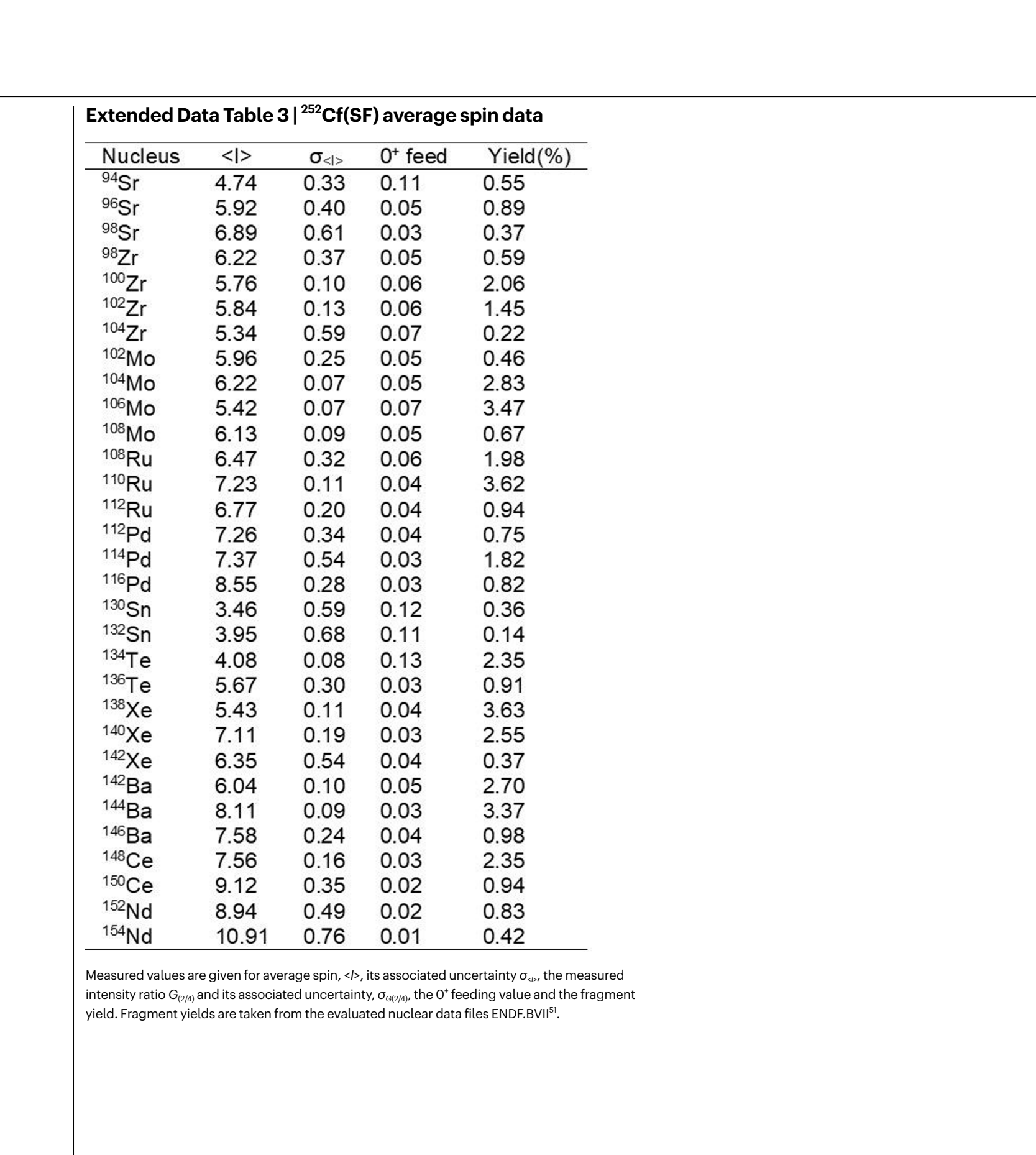




\section{Article}

Extended Data Table 4 | Fitting parameters for the light and heavy peak data for the three fissioning systems

\begin{tabular}{llll}
\hline System & Mass peak & Fitted constant $c$ & Correlation coefficient $R^{2}$ \\
\hline${ }^{232} \mathrm{Th}(\mathrm{n}, \mathrm{f})$ & light & 0.189 & 0.837 \\
${ }^{238} \mathrm{U}(\mathrm{n}, \mathrm{f})$ & light & 0.192 & 0.806 \\
${ }^{252} \mathrm{Cf}(\mathrm{SF})$ & light & 0.186 & 0.803 \\
${ }^{232} \mathrm{Th}(\mathrm{n}, \mathrm{f})$ & heavy & 0.195 & 0.848 \\
${ }^{238} \mathrm{U}(\mathrm{n}, \mathrm{f})$ & heavy & 0.202 & 0.854 \\
${ }^{252} \mathrm{Cf}(\mathrm{SF})$ & heavy & 0.213 & 0.884 \\
\hline
\end{tabular}

The fitting function is defined as $\langle I\rangle=\mathrm{c} A_{\mathrm{N}}^{1 / 4} A_{\mathrm{F}}^{7 / 12}$ with a single free parameter, $\mathrm{c}$. 


\section{Author Queries}

Journal: Nature

Paper: s41586-021-03304-w

Title: Angular momentum generation in nuclear fission

\section{AUTHOR:}

The following queries have arisen during the editing of your manuscript. Please answer by making the requisite corrections directly in thee.proofing tool rather than marking them up on thePDF. This will ensure that your correctionsare incorporated accurately and that your paper is published as quickly as possible.

\begin{tabular}{|c|c|}
\hline $\begin{array}{l}\text { Query } \\
\text { Reference }\end{array}$ & Reference \\
\hline Q1 & $\begin{array}{l}\text { Please check that the display items are as follows (ms no:2020-06-11306): Figs 1-4 (colour); Tables: None; } \\
\text { Boxes: None; Extended Data display items: } 4 \text { figs, } 4 \text { tables; SI: yes, just TPR. The eproof will contain the } \\
\text { main-text figures edited by us and the Extended Data items (unedited except for the legends) and the } \\
\text { Supplementary Information (unedited). Please check the edits to all main-text figures very carefully, } \\
\text { and ensure that any error bars in the figures are defined in the figure legends. If you need to revise any } \\
\text { Extended Data items please upload these files when you submit your corrections to the eproof. }\end{array}$ \\
\hline Q2 & $\begin{array}{l}\text { thank you for your affiliation corrections; the affiliations have been renumbered again into sequence; } \\
\text { please check carefully. }\end{array}$ \\
\hline Q3 & $\begin{array}{l}\text { Please check your article carefully, coordinate with any co-authors and enter all final edits clearly in the } \\
\text { eproof, remembering to save frequently. Once corrections are submitted, we cannot routinely make } \\
\text { further changes to the article. }\end{array}$ \\
\hline Q4 & $\begin{array}{l}\text { Note that the eproof should be amended in only one browser window at any one time; otherwise changes } \\
\text { will be overwritten. }\end{array}$ \\
\hline Q5 & $\begin{array}{l}\text { Author surnames have been highlighted. Please check these carefully and adjust if the first name or } \\
\text { surname is marked up incorrectly. Note that changes here will affect indexing of your article in public } \\
\text { repositories such as PubMed. Also, carefully check the spelling and numbering of all author names and } \\
\text { affiliations, and the corresponding email address(es). }\end{array}$ \\
\hline Q6 & $\begin{array}{l}\text { You cannot alter accepted Supplementary Information files except for critical changes to scientific } \\
\text { content. If you do resupply any files, please also provide a brief (but complete) list of changes. If these } \\
\text { are not considered scientific changes, any altered Supplementary files will not be used, only the } \\
\text { originally accepted version will be published. }\end{array}$ \\
\hline
\end{tabular}




\section{Author Queries}

Journal: Nature

Paper: s41586-021-03304-w

Title:Angular momentum generation in nuclear fission

\section{AUTHOR:}

The following queries have arisen during the editing of your manuscript. Please answer by making the requisite corrections directly in thee.proofing tool rather than marking them up on thePDF. This will ensure that your correctionsare incorporated accurately and that your paper is published as quickly as possible.

\begin{tabular}{|c|c|}
\hline $\begin{array}{l}\text { Query } \\
\text { Reference }\end{array}$ & Reference \\
\hline Q7 & $\begin{array}{l}\text { If applicable, please ensure that any accession codes and datasets whose DOIs or other identifiers are } \\
\text { mentioned in the paper are scheduled for public release as soon as possible, we recommend within a } \\
\text { few days of submitting your proof, and update the database record with publication details from this } \\
\text { article once available. }\end{array}$ \\
\hline Q8 & $\begin{array}{l}\text { [author: are the edits ok in "correlated with the indirect detection (plastic scintillator } 20 \text { and surface } \\
\text { barrier detectors21) of fragment mass"?] }\end{array}$ \\
\hline Q9 & $\begin{array}{l}\text { In the inset to Fig. 2a, I have now changed (a) to Pre-scission and (b) to Post-scission to be specific, ok? } \\
\text { If you still feel these labels could be confusing, we could change them to A and B? }\end{array}$ \\
\hline Q10 & $\begin{array}{l}\text { Please check whether any subheadings exceed one line of the PDF attached to the eproof, and shorten } \\
\text { as needed. }\end{array}$ \\
\hline Q11 & $\begin{array}{l}\text { Please note that our house style is to set scalar variables italic and to set vectors upright and boldface. } \\
\text { We do not use the over-arrow notation. }\end{array}$ \\
\hline Q12 & $\begin{array}{l}\text { I added this sentence "The superscripts are the exponents in the fitting function of equation (2); see } \\
\text { also Extended Data Fig. } 4 \text { " to clarify the exponents in Fig. } 4 \mathrm{a} \text { (and added numbers to the equations to } \\
\text { enable me to do so, ok? }\end{array}$ \\
\hline
\end{tabular}

